\title{
State-resolved $\mathrm{O}_{2}-\mathrm{N}_{2}$ kinetic model at hypersonic temperatures
}

\author{
Daniil A. Andrienko* \\ Iain D. Boyd ${ }^{\dagger}$ \\ Department of Aerospace Engineering, University of Michigan, Ann Arbor, MI, 48109
}

\begin{abstract}
A master equation simulation of $\mathrm{O}_{2}-\mathrm{N}_{2}$ thermal relaxation based on a new set of accurate rate coefficients is presented. Vibrationally resolved transitions rates are generated by means of the quasiclassical trajectory method at range of temperatures on ab-initio potential energy surface (PES). Verification of the QCT results using the experimental data in the wide temperature range between 300 and $6000 \mathrm{~K}$. The present model significantly overestimates the rates of energy transfer in collisions of vibrationally hot oxygen with cold nitrogen at room temperatures. At the same time, an excellent agreement with experimental data is obtained for vibrational relaxation times and dissociation rate coefficients at hypersonic temperatures.
\end{abstract}

\section{Nomenclature}

$v, w \quad$ initial vibrational states of $\mathrm{O}_{2}$ and $\mathrm{N}_{2}$

$v^{\prime}, w^{\prime} \quad$ final vibrational states of $\mathrm{O}_{2}$ and $\mathrm{N}_{2}$

$K \quad$ rate coefficient of bound-bound transition

$D, R \quad$ dissociation and recombination rate coefficients

$T, T_{r}, T_{v}$ translational, rotational and vibrational temperatures

\section{Introduction}

An important research direction in hypersonic aerothermochemistry is associated with the modeling of thermal and chemical nonequilibrium from first principles using quantum-mechanical, semi-classical and quasi-classical methods. The initial capacity for such research was formed by the ever-increasing computational power such that statistical methods became tractable as well as by the variety of potential energy surfaces (PES), developed for studies of low temperature kinetics in the upper atmosphere [1-3].

While a large amount of previous work regarding the nonequilibrium processes in high temperature gases has concentrated on the state-resolved chemistry of nitrogen [4-8] and nitric oxide [9-13] due to importance of these species in re-entry and combustion problems, thermochemistry of oxygen has received little attention. This is due to the fact that the atmosphere contains only 0.2 molar fraction of oxygen, and it dissociates easily under harsh re-entry conditions. However, recent success and setbacks of hypersonic programs have directed research in the study of oxygen nonequilibrium thermalization at lower speeds that correspond to the flight conditions of air-breathing hypersonic vehicles.

For diatomic oxygen, the most important collisions in terms of rovibrational relaxation and dissociation are observed with $\mathrm{O}, \mathrm{O}_{2}$ and, more importantly, with $\mathrm{N}_{2}$. Currently, only the first type of collisions studied in detail. For the $\mathrm{O}_{2}-\mathrm{O}$ system, a number of PESs [14-17] of different fidelity is available due to ability of $\mathrm{O}_{3}$ to absorb harmful ultra-violet radiation [18]. High-temperature kinetics of $\mathrm{O}_{2}-\mathrm{O}$ collisions received special attention recently due to anomalously high rate coefficients (RC) of vibrational transitions [19,20].

${ }^{*}$ Postdoctoral research fellow, Department of Aerospace Engineering, University of Michigan, 1320 Beal Ave

${ }^{\dagger}$ James E. Knott Professor, Department of Aerospace Engineering, University of Michigan, 1320 Beal Ave 
This phenomenon is associated with the small potential barrier in the entrance/exit channel of the $\mathrm{O}_{2}-\mathrm{O}$ interaction.

The study of collisions of molecular oxygen with other diatomic air species is limited to low temperatures (say, $1000 \mathrm{~K}$ ). There are a number of $\mathrm{O}_{2}-\mathrm{O}_{2}[1,21,22]$ and $\mathrm{O}_{2}-\mathrm{N}_{2}[2,23] \mathrm{PESs}$, however they are designed only for low energy collisions. In many cases, these PESs are either limited to a rigid rotor approximation, or utilize empirical data to approximate the intermolecular forces, or do not describe the potential energy in bond-breaking collisions. Nevertheless, the semi- and quasi-classical calculations, conducted on these PESs, showed good agreement with measurements in the rates of vibration-translation and vibration-vibration energy transfer between diatomic species at room temperatures [24]. The interest in such kinetic models is dictated by the disagreement in theoretical and experimental predictions of ozone formation rates [3], probably due to some shadow mechanisms involving vibrationally excited $\mathrm{O}_{2}[25,26]$.

Only recently the investigation of bimolecular collisions at higher temperatures received attention $[27,28]$ due to the availability of ab-initio four-body PESs. Six-dimensional $\mathrm{N}_{4}$ PESs based on accurate quantum calculations were developed at the NASA Ames Research Center [29] and at the University of Minnesota [27]. More interesting, in the context of the present paper, is the recent global $\mathrm{O}_{2} \mathrm{~N}_{2}$ PES by Truhlar and coworkers [28]. However, full-blown quasi-classical trajectory (QCT) simulations including all rovibrational states appears to be computationally intractable using these high-quality PESs. One of the possible ways to overcome this difficulty is the direct molecular simulation method, developed by Koura [30]. While this approach makes it possible to obtain the average macroscopic parameters of thermally and chemically nonequilibrium gas mixtures, such as vibrational and rotational relaxation times and the quasi-steady state (QSS) dissociation RC, this method does not allow extraction of the state-specific transition RCs that are typically required for further kinetic modeling in fluid dynamic codes and experiments.

The present paper features QCT calculations, conducted on the new $\mathrm{O}_{2} \mathrm{~N}_{2}$ PES and compares the global dissociation RCs and vibrational relaxation time with existing experimental data. The VT and VV energy transfer RC for selected states are compared with other semi-classical calculations as well. The limitations and applicability of the present technique is discussed. In Section II, the governing equations are given. Section III presents the master equation simulation of $\mathrm{O}_{2}-\mathrm{N}_{2}$ system. Conclusions are drawn in Section IV.

\section{Computational approach}

The quasi-classical trajectory method presents a compromise between the accuracy of statistical simulation of trajectory propagation and its cost. There are only a few alternatives to the QCT method when attempting to resolve all possible rovibrational state-specific cross sections at high kinetic energies, i.e. when inelastic collisions occur frequently, providing small statistical uncertainty of results.

The QCT method yields 12 and 18 Hamiltonian equations when applied to molecular systems with three (atom-diatom) and six (diatom-diatom) degrees of freedom. These numbers can be reduced to 8 and 14 by recognizing that the total energy and total angular momenta are conserved. On the other hand, the semiclassical (SC) coupled approach, proposed by Billing [31], involves additional time-dependent Schröedinger equations, whose number is proportional to the number vibrational states involved in a trajectory simulation. At hypersonic temperatures, when the population of all vibrational states must be captured, such a method becomes computationally intractable.

In the present work, the simulation of molecule-molecule collisions is conducted using an in-house fourbody QCT code. The extensive overview of three- and four-body QCT methods is given elsewhere [32]. The present paper concentrates on kinetic models of hypersonic flows and provides discussion on utilization of the QCT data. The primary tool for analysis of state-specific RCs, generated by the QCT method, is the system of master equations. The general appearance of the master equation for molecular oxygen includes the bound-bound and bound-free processes and is given by Eq. (1)

$$
\begin{array}{r}
\frac{d n_{v}}{d t}=\sum_{v^{\prime} \neq v, w^{\prime}, w} K_{\left(v^{\prime}, w^{\prime}\right) \rightarrow(v, w)} n_{w^{\prime}} n_{v^{\prime}}-\sum_{v \neq v^{\prime}, w, w^{\prime}} K_{(v, w) \rightarrow\left(v^{\prime}, w^{\prime}\right)} n_{w} n_{v}- \\
-\sum_{w, w^{\prime}} D_{(v, w) \rightarrow w^{\prime}} n_{v} n_{w}+\sum_{w, w^{\prime}} R_{w^{\prime} \rightarrow(v, w)} n_{x}^{2} n_{w^{\prime}}, \quad v=1 \ldots N_{v}
\end{array}
$$

where $n_{v}$ is the population of the $v$ vibrational state, $K_{\left(v^{\prime}, w^{\prime}\right) \rightarrow(v, w)}$ is the $\mathrm{RC}$ of reaction with $\mathrm{O}_{2}\left(v^{\prime}\right)$ and $\mathrm{N}_{2}\left(w^{\prime}\right)$ with the production of two molecular species in the $v$ and $w$ vibrational states, respectively. The RC 
of the reverse reaction is denoted as $K_{(v, w) \rightarrow\left(v^{\prime}, w^{\prime}\right)} \cdot n_{x}$ is the number density of the dissociation projectile, $D_{(v, w) \rightarrow w^{\prime}}$ and $R_{w^{\prime} \rightarrow(v, w)}$ are the state-specific dissociation and recombination rate coefficients of the $v$ state in collision with $\mathrm{N}_{2}(w)$ state with the production of $\mathrm{N}_{2}\left(w^{\prime}\right), N_{v}$ denotes the total number of vibrational states. The master equation for $\mathrm{N}_{2}(w)$ is similar to Eq. (1) with the only replacement of $v$ to $w, v^{\prime}$ to $w^{\prime}$ and vice versa.

Equation (1) describes the relaxation of molecular nitrogen and oxygen. In the present work the study of relaxation is restricted to the heat bath conditions. Initial conditions must be provided for the system of equations (1). Since the master equation simulation contains two molecules as reactants, initial conditions can be specified for each of them individually. In all simulations, the initial rotational temperature is set to the translational temperature. When vibrational relaxation is studied, the initial temperature of oxygen and nitrogen is set to either $100 \mathrm{~K}$ or to $10,000 \mathrm{~K}$, as specified. The first choice of the initial $T_{v}$ corresponds to the strong nonequilibrium conditions, while the second choice indicates that the given molecular species is in thermal equilibrium. The initial population of the vibrational ladder is calculated from the Boltzmann distribution at specified initial vibrational temperature. Although the production of nitric oxide is observed by means of the QCT method at the range of studied temperatures, the NO formation is of secondary importance for the thermal relaxation of $\mathrm{O}_{2}$ and $\mathrm{N}_{2}$ and, hence, is artificially excluded from the master equation simulation. To do so, the RCs of NO formation is set to zero and the master equations for the NO vibrational ladder are excluded from the system (1). The principle of detailed balance is invoked to generate rates of endothermic transitions, in order to reduce the statistical error of the QCT method.

The system of master equations is a convenient tool for the analysis of designated energy transfer mechanisms. Since the rate coefficients between vibrational states are of primary importance for $\mathrm{O}_{2}$ and $\mathrm{N}_{2}$ relaxation, in the present work, the result of $\mathrm{O}_{2}-\mathrm{N}_{2}$ collisions are divided into several categories. First, vibration-translation (VT) and vibration-vibration (VV) types of collisions are described by Eqs. (2)-(3) and by Eqs. (4)-(5), respectively:

$$
\begin{gathered}
\mathrm{O}_{2}(v)+\mathrm{N}_{2}(w) \rightarrow \mathrm{O}_{2}(v-\Delta v)+\mathrm{N}_{2}(w) \\
\mathrm{O}_{2}(v)+\mathrm{N}_{2}(w) \rightarrow \mathrm{O}_{2}(v)+\mathrm{N}_{2}(w-\Delta w) \\
\mathrm{O}_{2}(v)+\mathrm{N}_{2}(w) \rightarrow \mathrm{O}_{2}(v-\Delta v)+\mathrm{N}_{2}(w-\Delta w) \\
\mathrm{O}_{2}(v)+\mathrm{N}_{2}(w) \rightarrow \mathrm{O}_{2}(v-\Delta v)+\mathrm{N}_{2}(w-\Delta w)
\end{gathered}
$$

The reactions of depletion can be divided into single and double dissociation event categories:

$$
\begin{gathered}
\mathrm{O}_{2}(v)+\mathrm{N}_{2}(w) \rightarrow \mathrm{O}+\mathrm{O}+\mathrm{N}_{2}(w-\Delta w) \\
\mathrm{O}_{2}(v)+\mathrm{N}_{2}(w) \rightarrow \mathrm{O}_{2}(v-\Delta v)+\mathrm{N}+\mathrm{N} \\
\mathrm{O}_{2}(v)+\mathrm{N}_{2}(w) \rightarrow \mathrm{O}+\mathrm{O}+\mathrm{N}+\mathrm{N}
\end{gathered}
$$

The approach for obtaining rate coefficients for reactions Eqs. (2) - (8) from the database of cross sections was described previously elsewhere [33]. The e-folding method by Park [34] is adopted to extract the vibrational relaxation time from the temporal evolution of $\mathrm{O}_{2}$ and $\mathrm{N}_{2}$ vibrational energy. This method is adopted throughout the paper unless stated otherwise. A simpler approach to obtain the vibrational relaxation time is based on the rate coefficient of a monoquantum deactivation from the first excited vibrational state [35]. This approach is adopted in the present paper as an alternative to the e-folding method.

\section{Results}

The result section is organized as follows. First, RCs of VV and VT energy transfer for vibrationally excited oxygen are compared with the experimental data collected at $300 \mathrm{~K}$. Second, the RC of energy transfer between the first excited and ground vibrational states of oxygen is compared to the theoretical data obtained on another PES in the range of temperatures between 1000 and $7000 \mathrm{~K}$. The vibrational relaxation times and equilibrium dissociation $\mathrm{RC}$ are compared to the experimental data collected from shock tube measurements. These results are followed by the master equation simulation.

The interaction of $\mathrm{O}_{2}$ with molecular nitrogen is of great importance in hypersonic thermochemistry due to the large difference in the dissociation energy of these species. There are several PESs in the literature that describe the $\mathrm{N}_{2} \mathrm{O}_{2}$ system. One of the first $\mathrm{N}_{2} \mathrm{O}_{2}$ PESs was proposed by Aquilanti et al. [2] for studying rigid 
molecules in their ground vibrational state. This PES was designed specifically for methods of molecular dynamics for the upper atmosphere, however it has a little use for aerothermodynamics. Bartolomei et al. [36] recently published an improved ab-initio PES with similar restrictions. The vibration-translation (VT) and vibration-vibration (VV) energy transfer was studied by a semi-classical coupled method by Billing on an empirical PES in the range of temperatures between 250 and $1000 \mathrm{~K}$ [23]. Recently, Garcia et al. [37] reported VT and VV RCs using an improved PES for selected vibrational states of oxygen at temperatures up to $7000 \mathrm{~K}$. This PES is based on ab-initio calculations that are interpreted as a combination of van der Waals and electrostatic interaction terms. Unfortunately, the dataset reported in Ref. [37] is incomplete and, thus, a master equation simulation still can not be performed.

An analytical fit to the global ab-initio PES of triplet $\mathrm{N}_{2} \mathrm{O}_{2}$ was recently published by Truhlar's group. [28] This PES was obtained by multi-state complete-active space second-order perturbation theory and was specifically designed to describe collisions at high kinetic energies. Most of the multireference calculations of electron correlation energy were conducted in the range of potential energies between 100 and $350 \mathrm{kcal} / \mathrm{mole}$ ( $76 \%$ of the total 54,889 data points). It is worth to note that the long-range forces are not described correctly in this PES, thus it may not be suitable for low energy collisions.

\section{A. Vibrational energy exchange at low temperatures}

The vibrational quenching of oxygen in collisions with $\mathrm{N}_{2}$ is an important mechanism of energy transfer not only at hypersonic temperatures [38] but also for the chemistry of the upper atmosphere. Park and Slanger [24] have measured the rates of double-quantum quenching of oxygen via the VV energy transfer at room temperature:

$$
\mathrm{O}_{2}(v)+\mathrm{N}_{2}(w=0) \rightarrow \mathrm{O}_{2}(v-2)+\mathrm{N}_{2}(w=1) .
$$

The process, described by Eq. (9) is resonant at $v=18 / 19$ with the energy increment of 24 and -24 $\mathrm{cm}^{-1}$, respectively. The single quantum quenching has a large discrepancy of vibrational energy increment: -1165 and $-1189 \mathrm{~cm}^{-1}$. Overall, the rate coefficients of reaction (9) were obtained for $v=8 \ldots 22$ in [24]. The resonance of (9) led to a conclusion that this process can not be responsible for the rapid production of ozone in the atmosphere, as was previously prosed by Slanger et al. [39]. The experimental data from [24] still can be used to determine the range of applicability of the new $\mathrm{O}_{2} \mathrm{~N}_{2}$ PES.

The RCs of VV and VT energy transfer were previously computed by Billing [23] using the semi classical trajectory propagation on an empirical PES. Besides the RC of the double-quantum VV energy transfer reaction, given by Eq. (9), the rates of the following reactions were obtained:

$$
\begin{aligned}
& \mathrm{O}_{2}(v)+\mathrm{N}_{2}(w=0) \rightarrow \mathrm{O}_{2}(v-1)+\mathrm{N}_{2}(w=1), \\
& \mathrm{O}_{2}(v)+\mathrm{N}_{2}(w=0) \rightarrow \mathrm{O}_{2}(v-2)+\mathrm{N}_{2}(w=0), \\
& \mathrm{O}_{2}(v)+\mathrm{N}_{2}(w=0) \rightarrow \mathrm{O}_{2}(v-1)+\mathrm{N}_{2}(w=0) .
\end{aligned}
$$

In Fig. (1), the rate coefficients of reactions (9), (10) and (12) are presented. The circular symbols with the curve fit, shown by the red line, presented the RC of reaction (9) obtained in the experimental work by Park and Slanger [24]. Black dashed and solid lines describe the RCs of reactions (9) and (12), obtained by Billing. The square, circular and diamond symbols correspond to the present RCs of reactions (12), (10) and (9) at $v=15 \ldots 24$, respectively.

Detailed comparison of RCs at $\mathrm{T}=300 \mathrm{~K}$ is given in Table 1. The present $\mathrm{QCT}$ calculations suggest that the singlequantum VT mechanism is the most probable path for the energy transfer. Overall, the singlequantum VV and VT reactions have higher RCs than of the double-quantum reactions. On the other hand, the results by Billing indicate that the resonant double-quantum VV transition and single-quantum VT transitions are the most probable ones. Such drastic differences between the present results and Billing work should be attributed to the difference in PESs. Indeed, in the original work by Varga et al. [28], the following is stated: "We emphasize that the present surface was designed for high-energy collisions, and one should expect that it does not have the correct long-range weak interactions that can be very important for low-temperature simulations". Indeed, a stronger long-range attraction in Varga's PES compared to the PES by Billing may smear the effect of resonance in the double-quantum transition.

Slices of these two PESs for several selected locations of the oxygen and nitrogen molecules are shown in Fig. 2. In these calculations, parameters for the Billing PES are taken from the original work [23]. The 


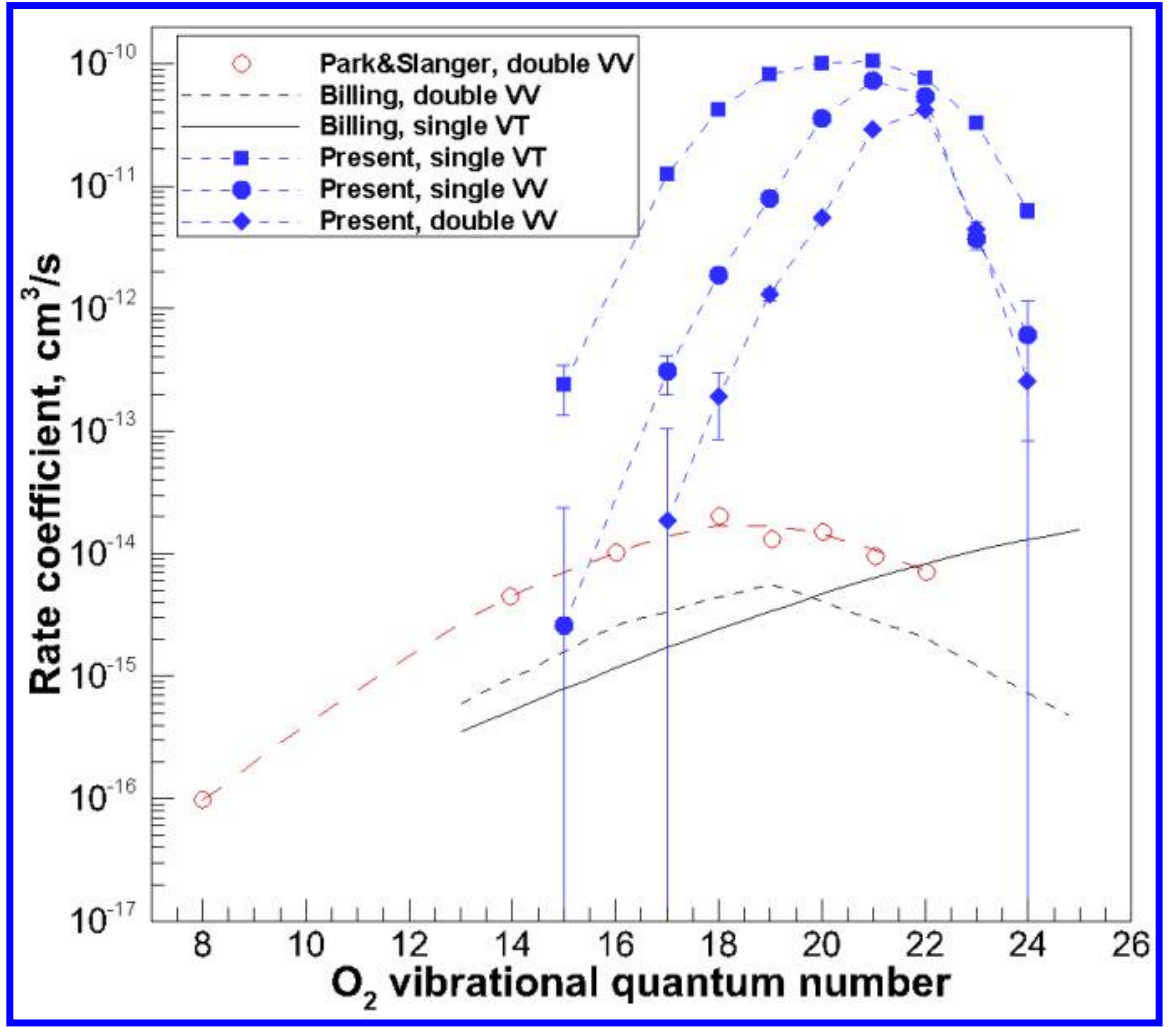

Fig. 1: Rate coefficients of single-quantum VT and double-quantum energy exchange reactions

\begin{tabular}{ccccc} 
Reaction & Single, VV & Double, VV & Single, VT & Double, VT \\
\hline Present & $7.87 \times 10^{-12}$ & $1.31 \times 10^{-12}$ & $8.19 \times 10^{-11}$ & $7.54 \times 10^{-29}$ \\
Billing & $1.4 \times 10^{-20}$ & $5.1 \times 10^{-15}$ & $3.3 \times 10^{-15}$ & $1.5 \times 10^{-17}$
\end{tabular}

Table 1: $\mathrm{O}_{2}(v=19)-\mathrm{N}_{2}(w=0) \mathrm{VV}$ and VT transition $\mathrm{RCs}\left[\mathrm{cm}^{3} / \mathrm{s}\right]$ at $T=T_{v}=T_{r}=300 \mathrm{~K}$

abscissa corresponds to the distance between $\mathrm{O}_{2}$ and $\mathrm{N}_{2}$ center of mass. Potential is given in meV. For all studied configurations, the PES developed by Varga et al. demonstrate a substantially deeper potential well, compared to that of Billing PES. This difference is $3 \mathrm{meV}$ for the L configuration (collinear approach). For the $\mathrm{X}$ configuration, the difference is approximately $10 \mathrm{meV}$ which is comparable with the energy increment during a collision with VV energy transfer. Besides the differences in depth of potential well, Varga's PES has a less repulsive potential wall.

Meanwhile, the RCs of VV and VT energy transfer for $\mathrm{O}_{2}(v=25)$ were computed by Garcia et al. [37] on two PESs of different fidelity utilizing the SC and QCT methods. In the present work, rate coefficients in Ref. [37] are compared with those obtained by the QCT method on the PES of Varga et al. [28]. The VT reaction corresponds to the mono- and double-quantum deactivation of oxygen with nitrogen remaining in the same vibrational state. The $\mathrm{VV}$ reaction is described by $\mathrm{O}_{2}$ mono-quantum deactivation with the simultaneous single quantum activation of nitrogen. Rate coefficients of these processes are shown in Table 2.

The present QCT results agree better with the data, obtained on the Garcia PES based on ab-initio data, than with the data generated using the simpler Billing PES. This is observed for all three types of considered transitions. In fact, the Billing PES produces a systematic overestimation of rates, which may be explained by the fact that this PES is more repulsive than the Garcia ab-initio PES, and the attraction in the $\mathrm{N}_{2} \mathrm{O}_{2}$ dimer configuration in the Billing PES is less pronounced [37]. As expected, the rates of VT transition, generated in the present work, generally agree better with the results of QCT calculations than with the SC method.

The situation of energy transfer in collisions with oxygen in the ground vibrational states is drastically 


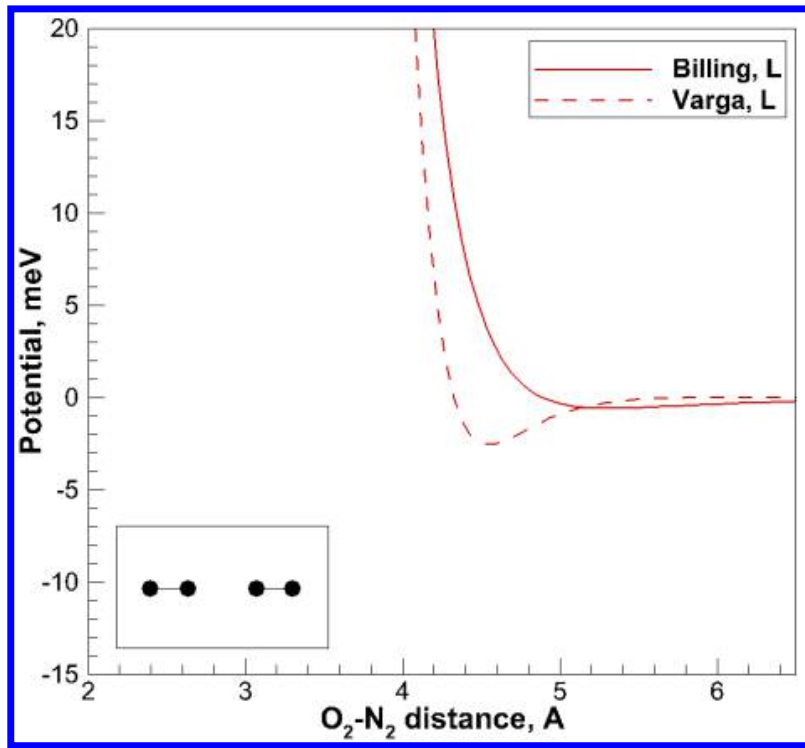

(a) L configuration

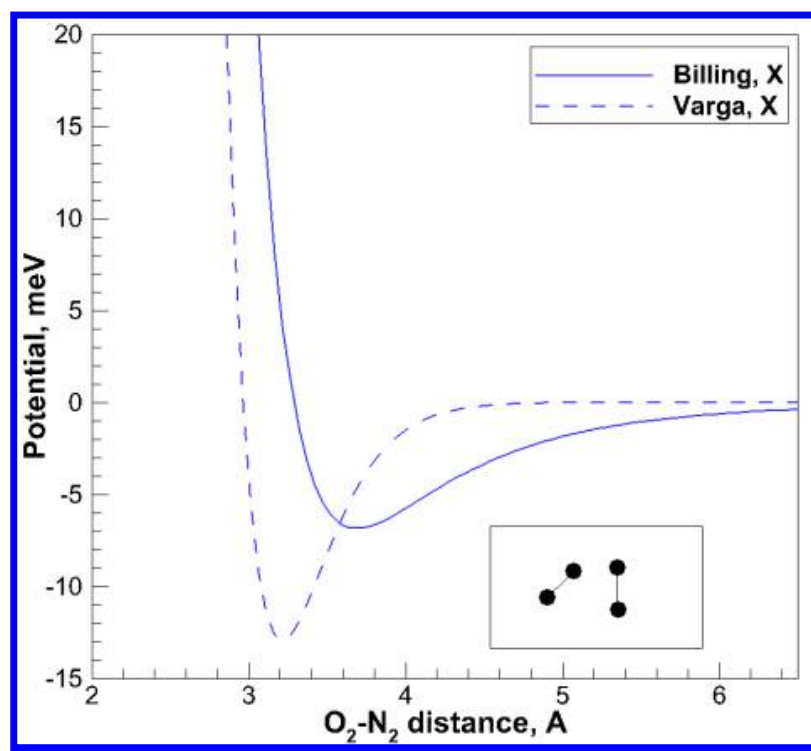

(c) X configuration

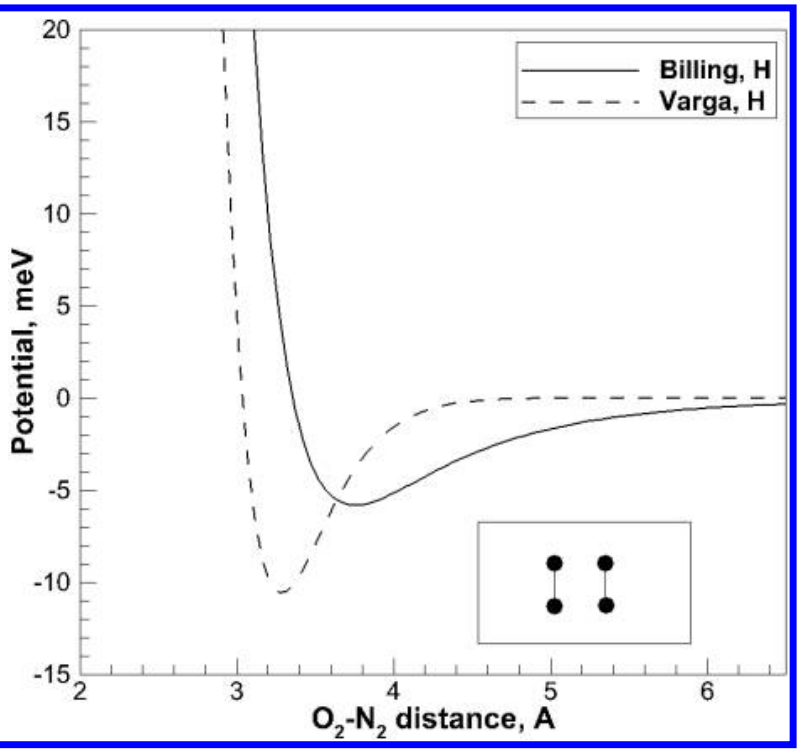

(b) $\mathrm{H}$ configuration

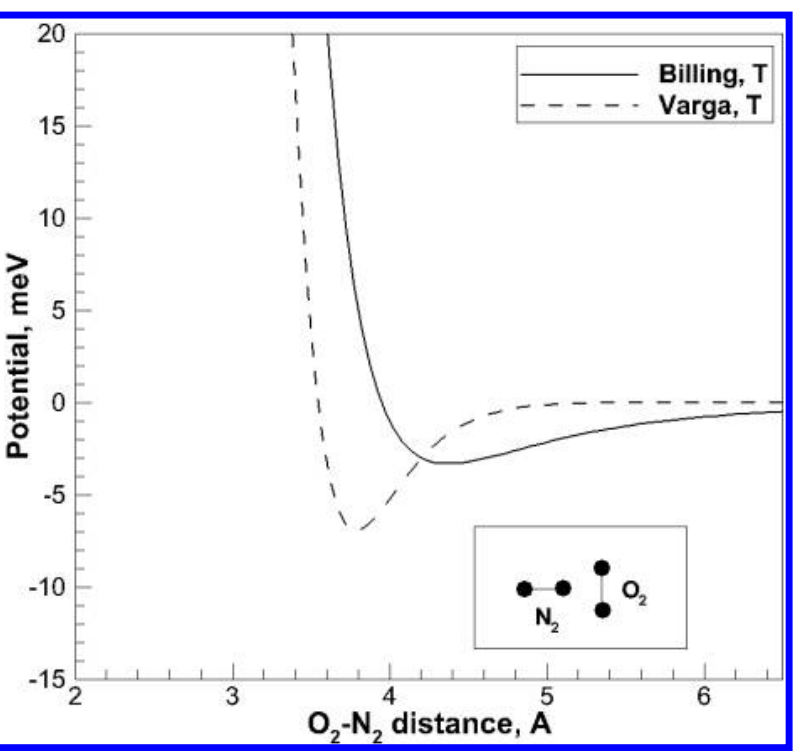

(d) $\mathrm{T}$ configuration

Fig. 2: Comparison of Billing [23] and Varga et al. [28] $\mathrm{O}_{2}-\mathrm{N}_{2}$ PESs 


\begin{tabular}{ccc} 
Source & Transition & Rate coefficient \\
\hline QCT, Present & $\mathrm{O}_{2}(v-1)+\mathrm{N}_{2}(w)$ & $4.36 \times 10^{-12}$ \\
QCT, Garcia PES [37] & - & $2.3 \times 10^{-12}$ \\
SC, Garcia PES [37] & - & $1.1 \times 10^{-12}$ \\
QCT, Billing PES [23] & - & $1.2 \times 10^{-11}$ \\
SC, Billing PES [23] & - & $3.5 \times 10^{-12}$ \\
\hline QCT, Present & $\mathrm{O}_{2}(v-2)+\mathrm{N}_{2}(w)$ & $8.16 \times 10^{-14}$ \\
QCT, Garcia PES [37] & - & $1.1 \times 10^{-13}$ \\
SC, Garcia PES [37] & - & $6.0 \times 10^{-14}$ \\
QCT, Billing PES [23] & - & $1.5 \times 10^{-12}$ \\
SC, Billing PES [23] & - & $3.9 \times 10^{-13}$ \\
\hline QCT, Present & $\mathrm{O}_{2}(v-1)+\mathrm{N}_{2}(w+1)$ & $2.17 \times 10^{-15}$ \\
SC, Garcia PES [37] & - & $1.6 \times 10^{-17}$ \\
SC, Billing PES [23] & - & $3.7 \times 10^{-16}$
\end{tabular}

Table 2: $\mathrm{O}_{2}(v=25)-\mathrm{N}_{2} \mathrm{VV}$ and VT transition $\mathrm{RCs}\left[\mathrm{cm}^{3} / \mathrm{s}\right]$ at $T=T_{r}=1000 \mathrm{~K}$

different. The VT and VV rates of energy exchange in $\mathrm{O}_{2}(v=0)+\mathrm{N}_{2}(w=1)$ are shown in Fig. 3. VV RCs correspond to the products $\mathrm{O}_{2}\left(v^{\prime}=1\right)+\mathrm{N}_{2}\left(w^{\prime}=0\right)$ while the VT RCs describe the production of $\mathrm{O}_{2}\left(v^{\prime}=0\right)+\mathrm{N}_{2}\left(w^{\prime}=0\right)$. Results of SC calculations by Garcia et al. [37] are shown by empty symbols. An empirical rate of VV transition, derived by Gilmore et al. [40] from experimental data on transition probabilities of the VV exchange reaction $[38,41]$ is shown by the long dashed curve. The experimental approach combines generation of spark interferograms with the following analysis of density profiles as well as the measurements of infrared emission from $\mathrm{CO}$ at $4.6 \mu$ of wavelength, used as a tracer for the $\mathrm{N}_{2}$ population. The last fact can be justified taking into account the very close fundamental vibrational frequencies of $\mathrm{N}_{2}$ and $\mathrm{CO}$.

Two important conclusions can be derived from this comparison. First, the agreement between the SC and QCT approaches is satisfactory only at temperatures higher than $3000 \mathrm{~K}$. Below this temperature, the QCT method strongly underestimates the RC of VV and VT transitions, compared to the result of SC calculations. There are two possible explanations for this disagreement. First, the QCT and SC calculations were conducted on two different $\mathrm{O}_{2} \mathrm{~N}_{2}$ PESs. Although the difference between these two methods in Table 2 is not as large as for the RCs involving $\mathrm{O}_{2}(v=0)$ in Fig. 3, only SC calculations on the PES given in [28] could provide an explanation for this difference.

Second, there is a possibility for the failure of the QCT method at low temperatures due to a pronounced influence of quantum effects. Previously it was shown that the QCT approach severely underestimates the $\mathrm{RC}$ of $\mathrm{O}_{2}(v=1)$ monoquantum deactivation in collisions with $\mathrm{Ar}$ at hypersonic temperatures [42]. These differences can not be explained by statistical uncertainty of the results. Similarly to $\mathrm{O}_{2}-\mathrm{Ar}$, the QCT method in the present work underestimates selected RCs compared to the SC method that adopts a quantal treatment of the vibrational mode.

Another important observation is related to the disagreement in VV RCs between the SC method and experimental data. This may be attributed to some uncertainties in the PES, used in [37], as well as to the theoretical interpretation of the experimental data. Indeed, the analysis of interferograms in Ref. [38] and [41] was outlined assuming that the relaxation of nitrogen in the $\mathrm{O}_{2}-\mathrm{N}_{2}$ mixture can be represented as a superposition of two processes with different time scales. During a short period of time after the shock passage, oxygen quickly relaxes in collisions with $\mathrm{N}_{2}$ and $\mathrm{O}_{2}$ molecules via the VT energy transfer mechanism. After molecular oxygen receives a sufficient amount of vibrational energy from the translational motion of the particles, the energy exchange between $\mathrm{N}_{2}$ and $\mathrm{O}_{2}$ starts to play a role. Moreover, in experimental work by White [38] it was assumed that the VT process of $\mathrm{N}_{2}$ excitation in collisions with oxygen is noticeably slower than the $\mathrm{N}_{2}-\mathrm{O}_{2} \mathrm{VV}$ process, and the latter process should be responsible for the rapid excitation of $\mathrm{N}_{2}$ in air relative to pure $\mathrm{N}_{2}$.

The experimental data on vibrational relaxation time in pure oxygen is well known [43] and can be interpreted in terms of the monoquantum deactivation $\mathrm{RC}$ from $\mathrm{O}_{2}(v=1)$. Such data is shown in Fig. 3 


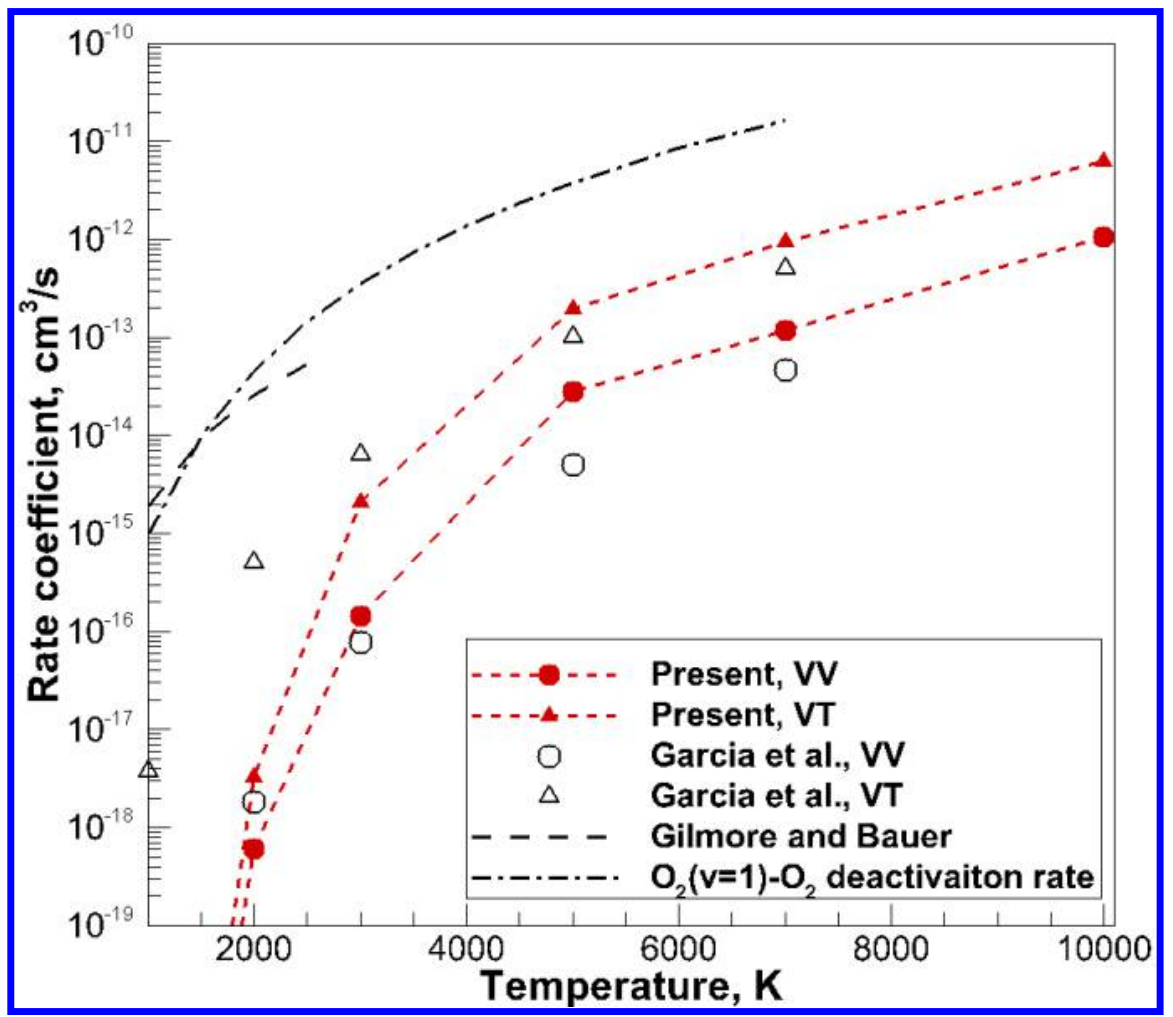

Fig. 3: Transition RCs of $\mathrm{O}_{2}(v=0)+\mathrm{N}_{2}(w=1)$ collision

with a dashed-dotted line. Indeed, the RC of monoquantum deactivation in pure $\mathrm{O}_{2}$ is substantially higher than either VT or VV RCs in $\mathrm{O}_{2}(v=0)+\mathrm{N}_{2}(w=1)$ collisions obtained via SC and QCT methods. However the RCs of $\mathrm{O}_{2}-\mathrm{N}_{2} \mathrm{VV}$ transition by Gilmore et al. [40] are comparable to the relaxation RC in pure oxygen and do not comply with the hypothesis about the two stage $\mathrm{N}_{2}$ relaxation process in air [38]. Moreover, the $\mathrm{RC}$ of the VT process, given by empty and filled triangles, is higher than the RC of the VV process obtained via the trajectory simulation approach. This indicates that the $\mathrm{MW}$ correlation rule for $\mathrm{N}_{2}-\mathrm{O}_{2}$ collisions, used in [38] to solve for the VV exchange RC, may not be accurate enough for the analysis of the experimental data. Clearly, the most rigorous approach would consist of analysis of interferograms, obtained in [38,41] using the new RCs obtained on accurate PES by means of trajectory propagation methods accounting for quantum effects.

\section{B. VT and VV energy transfer at high temperatures}

The solution of master equations provides important information about specific populations of the rovibrational manifold as well as about general macroscopic properties of the evolving non-equilibrium system, such as internal temperatures, global and QSS dissociation RCs and thermal relaxation times. In order to extract this data, a complete set of VT and, sometimes, VV rates for each combination of $\mathrm{O}_{2}(v)$ and $\mathrm{N}_{2}(w)$ vibrational states must be available. Because QCT simulations of bi-molecular systems are expensive, the adopted approach obtains RCs for selected vibrational states and interpolates the rest of data. The computational feasibility of such approach for $\mathrm{O}_{2}-\mathrm{N}_{2}$ was demonstrated by Garcia [37].

The QCT method can be used for comparison of vibrational relaxation time, derived from the rate of monoquantum deactivation from the first excited vibrational state with existing experimental data. The vibrational relaxation time is shown in Fig. 4. The dashed line corresponds to the result by the MillikanWhite equation with parameters estimated from the vibrational frequency of oxygen and the reduced mass of colliding particles. The MW curve is not obtained by fitting the general expression to experimental results. Circular symbols correspond to $\mathrm{O}_{2}$ vibrational relaxation times measured in air and in a $5 \% \mathrm{O}_{2}-95 \% \mathrm{~N}_{2}$ mixture by Generalov and Losev. The red line describes the present $\mathrm{O}_{2}$ relaxation time obtained on the PES of Truhlar's group. 


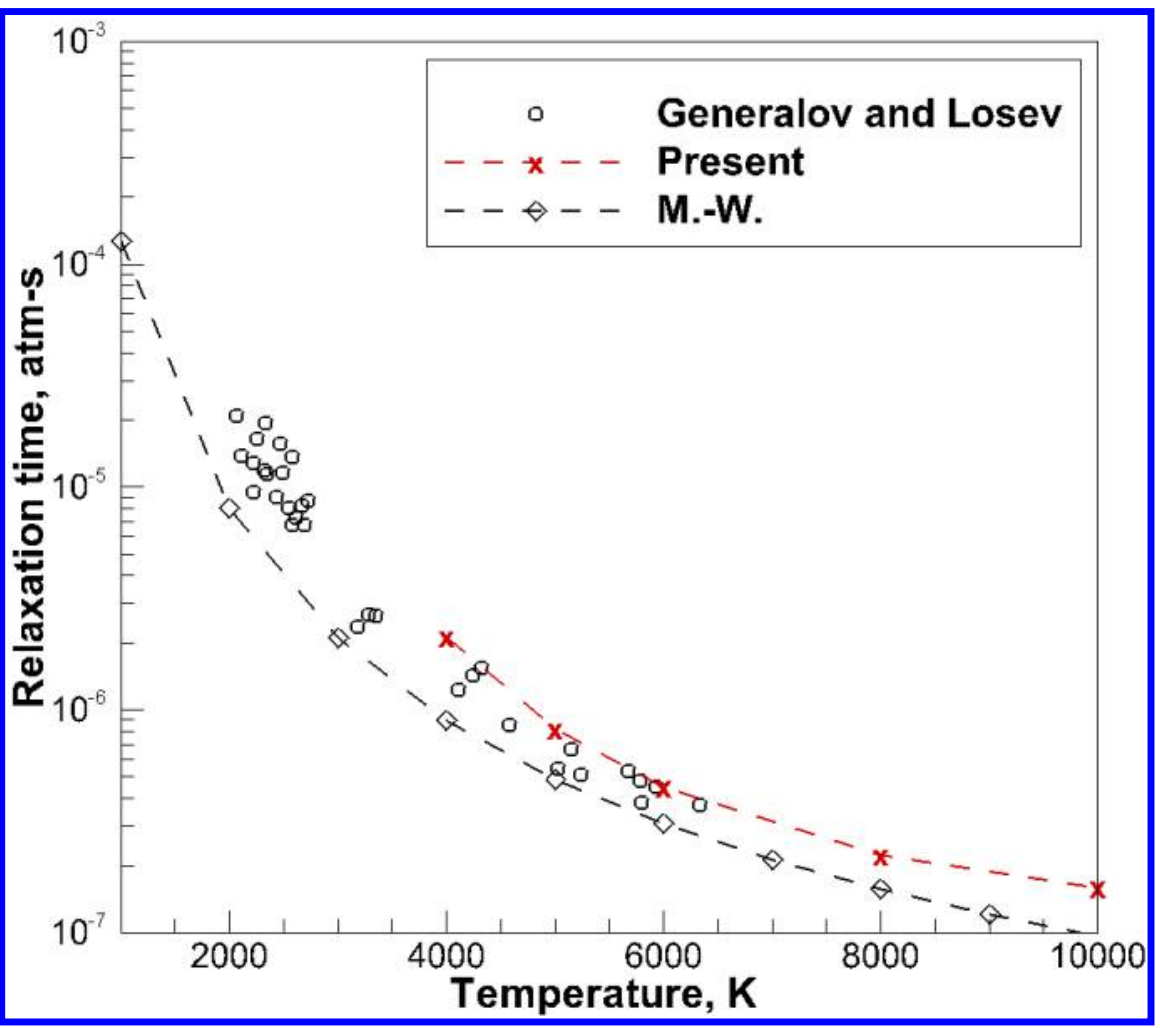

Fig. 4: Vibrational relaxation time of oxygen in collisions with $\mathrm{N}_{2}, T=T_{r}$

The experimental data by Generalov and Losev indicates a slower vibrational relaxation time of oxygen in collisions with $\mathrm{N}_{2}$, compared to the empirical equation by Millikan and White. This overestimation is very modest and, probably, falls in the uncertainty interval of $15-30 \%$ as stated in the original work [44]. More importantly, the present QCT results indicate better agreement with the data by Generalov and Losev rather than with the MW equation. At higher temperatures, namely at 10,000 K, it is possible to see the larger deviation of the present relaxation times from the MW expression. This is due to the increased contribution of multiquantum transitions and relaxation of highly excited vibrational states.

The dissociation rate of oxygen in collision with molecular nitrogen was measured previously in shock tube facilities [44-46]. In the work by Generalov and Losev [44] the rate of dissociation was derived from the initial slope of $\mathrm{O}_{2}$ concentration after the quasi-stationary distribution was obtained. The rate of oxygen depletion was analyzed by absorption in the SR bands at the wavelength of $224.5 \mathrm{~nm}$. This data is limited to the temperature range between 2700 and $7000 \mathrm{~K}$. In the more recent work by Jerig et al. [46], the rate of dissociation was measured by means of atomic resonance absorption spectroscopy (ARAS) in the range of temperatures between 2400 and $4100 \mathrm{~K}$. This method allows relatively accurate measurements of atomic oxygen concentration in the range of number densities between $10^{12}-10^{14} \mathrm{~cm}^{-3}$ at a wavelength of $130.5 \mathrm{~nm}$.

The thermal equilibrium dissociation RC of oxygen is shown in Fig. 5. Circular symbols describe the present QCT data, the dashed line extrapolates the QCT $D^{e q}$ at temperatures below $6000 \mathrm{~K}$. Filled symbols correspond to $D^{e q}$ obtained from the experimental measurements of $D^{Q S S}$ [44-46] by means of Eq. (??). The present extrapolated results are in very good agreement with the measurements by Jerig et al. [46]. The shock tube measurements by Generalov and Losev have much larger scatter, however the QCT RC is well within the uncertainty range. For extrapolation, the generalized Arrhenius form is used. The corresponding coefficients for $\mathrm{O}_{2}-\mathrm{N}_{2}$ thermal equilibrium dissociation $\mathrm{RC}$ are found to be $A=8.132 \times 10^{-10} \mathrm{~cm}^{3} / \mathrm{s}, B=-0.131$, $C=59380 \mathrm{~K}$. This expression is valid for translational temperatures between 2000 and $10,000 \mathrm{~K}$. 


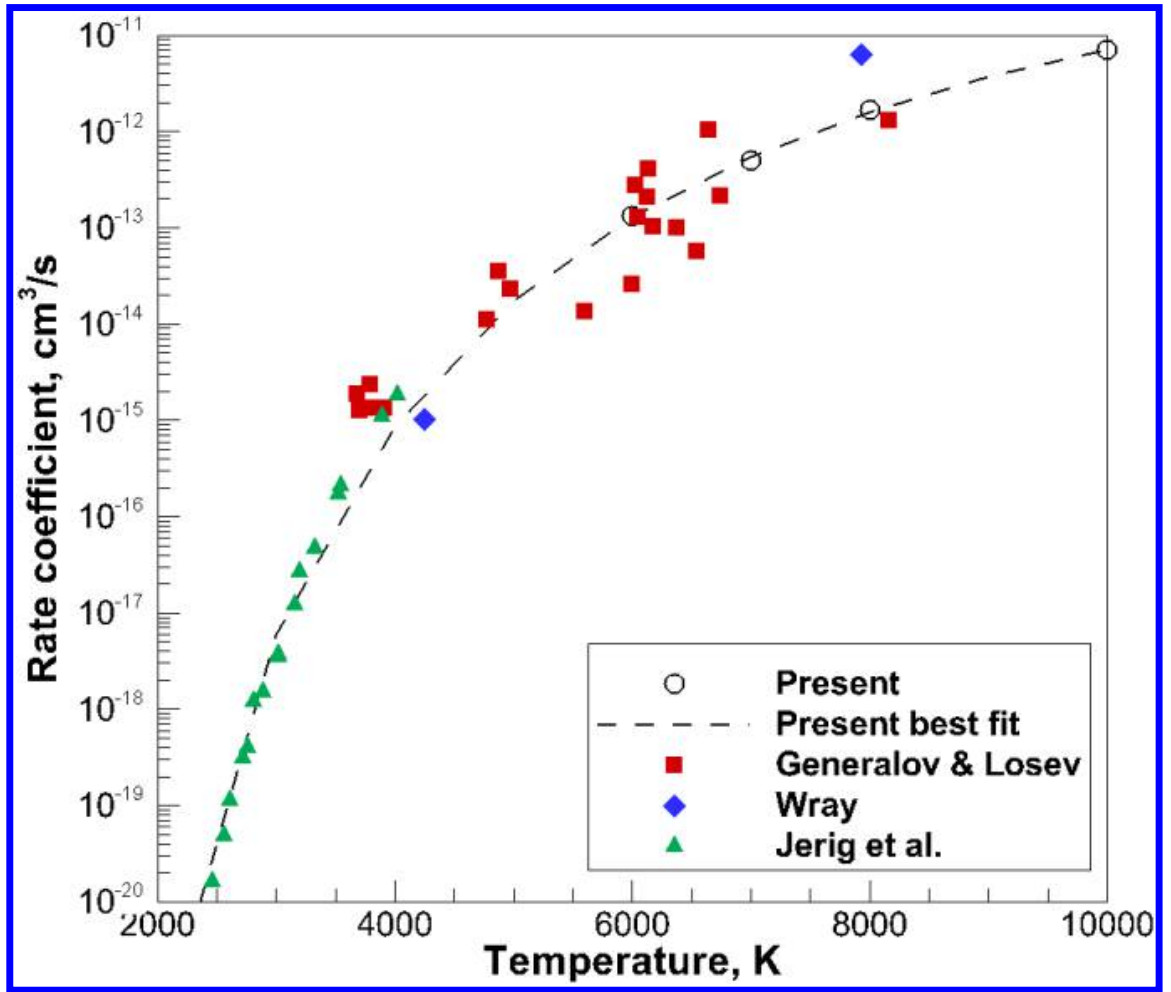

Fig. 5: Thermal equilibrium dissociation $\mathrm{RC}$ of oxygen in collisions with $\mathrm{N}_{2}$

\section{Full state-resolved results}

The simulation of heat bath conditions by means of a master equation requires a complete set of transitions RCs for all possible combinations of initial and final states. An assumption of trans-rotational equilibrium allows a significant reduction of the database, however, the number of possible transitions between vibrational states remains large. To overcome this difficulty, the present QCT simulations have been conducted for selected vibrational states of oxygen and nitrogen, and the missing RCs are interpolated using the QCT data. Namely, the $\mathrm{O}_{2}(v)$ RCs with $v$ chosen from $\Omega_{v}=\{0,1,2,5,10,15,20,25,30,31,32,33,34,35\}$ and for $\mathrm{N}_{2}(w)$ with $w$ chosen from $\Omega_{w}=\{0,1,2,5,10,15,20,25,30,35,40,45,50,51,52,53\}$ are obtained by the QCT method.

Because the RCs demonstrate a complex dependence on vibrational energy, as follows from Fig. 1, a global form of such dependence for bound-bound transition RCs is not derived. Instead, a liner interpolation of RCs is applied with the initial vibrational energy of reactant as an independent variable. First, the RCs are interpolated for all $\mathrm{O}_{2}$ initial vibrational states $v^{\star}$ at $\mathrm{N}_{2}$ initial vibrational $w$ from $\Omega_{w}$ :

$$
K\left(v^{\star}, w\right)=\frac{e_{v}^{\star}-e_{v}^{L}}{e_{v}^{R}-e_{v}^{L}} K\left(v^{R}, w\right)+\frac{e_{v}^{R}-e_{v}^{\star}}{e_{v}^{R}-e_{v}^{L}} K\left(v^{L}, w\right),
$$

where indices $\mathrm{R}$ and $\mathrm{L}$ correspond to the vibrational quantum numbers from $\Omega_{v}$ that have higher and lower vibrational energy than the state $v^{\star}$. No interpolation is required for the first three and for the last five vibrational states of oxygen since the QCT calculations are performed for those $v$. Following this procedure, RCs are evaluated for all initial $v$ and for $w$ from $\Omega_{w}$. Now, a similar algorithm is applied to interpolate RCs for all initial $w$. The following equation completes the interpolation procedure:

$$
K\left(v, w^{\star}\right)=\frac{e_{w}^{\star}-e_{w}^{L}}{e_{w}^{R}-e_{w}^{L}} K\left(v, w^{R}\right)+\frac{e_{w}^{R}-e_{w}^{\star}}{e_{w}^{R}-e_{w}^{L}} K\left(v, w^{L}\right) .
$$

The interpolation of dissociation RCs is conducted in a similar manner, only in this case, the linear interpolation is conducted for the natural logarithm of the RC. The independent variable, vibrational energy, is kept the same. 
The QCT RCs of $\mathrm{N}_{2}$ mono- and multi-quantum transitions in VT collisions with molecular oxygen are shown in Figs. $6 \mathrm{a}-6 \mathrm{c}$. The initial vibrational state of oxygen is set to $v=0$ in Fig. $6 \mathrm{a}$, to $v=10$ in Fig. $6 \mathrm{~b}$ and to $v=20$ in Fig. 6c. For reference, the corresponding FHO [47] RCs at $T=10,000 \mathrm{~K}$ are shown in Fig. 6a with the dashed lines. The FHO RCs demonstrate similarity to those of the QCT simulation; however, several differences persist. The monoquantum FHO RC is smaller than the QCT RC at any $\mathrm{N}_{2}$ initial vibrational energy with a maximal difference of a factor of 2.5 times.

For multiquatum VT transitions, the FHO rates generally repeat the shape of the QCT RCs with one distinct difference that the QCT rates decrease slower than those predicted by the FHO model at the $\mathrm{N}_{2}$ dissociation limit. Overall, the $\mathrm{RC}$ of $\mathrm{N}_{2}$ vibrational deactivation decreases rapidly with the increase of $\Delta w$. This is true for low-lying vibrational states of nitrogen due to small anharmonicity in those states. For highly excited nitrogen the RCs of multi- and single-quantum jumps become comparable, first of all because of the smaller spacing between these states. As will be shown shortly, the multiquantum jumps are of a great importance for the kinetics of highly-lying $\mathrm{N}_{2}$ vibrational states. The present QCT VT RCs demonstrate a small variation with respect to $\mathrm{O}_{2}$ vibrational state which is expected for the strictly vibration-translation energy transfer mechanism.

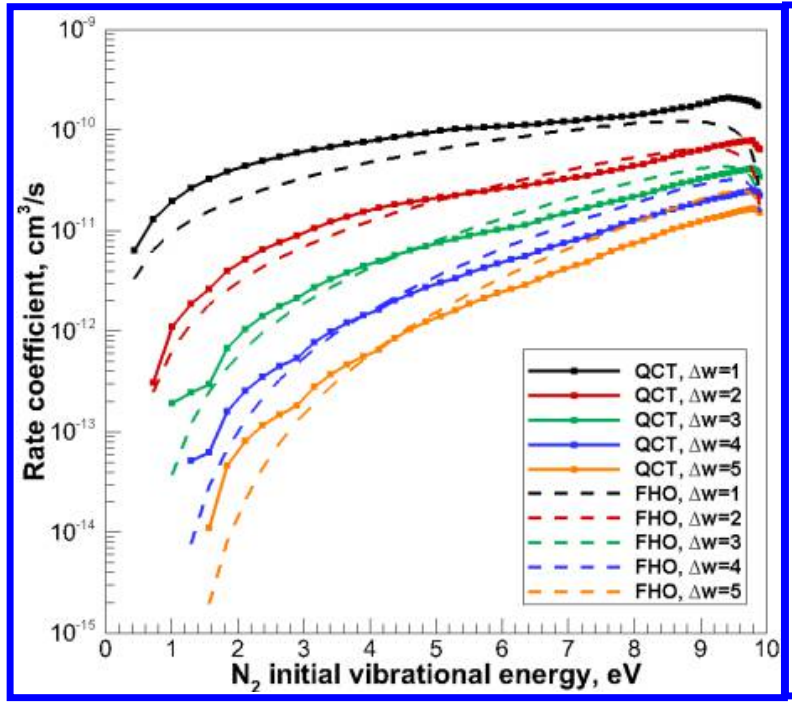

(a) QCT with $\mathrm{O}_{2}(v=0)$ and $\mathrm{FHO}$ with $\mathrm{T}=10,000 \mathrm{~K}$

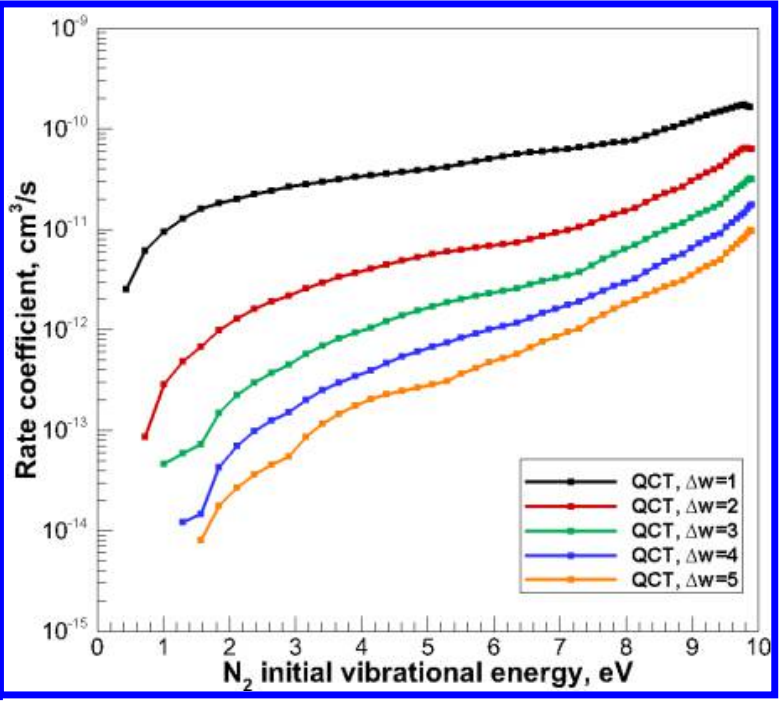

(b) $\mathrm{QCT}, \mathrm{O}_{2}(v=10)$

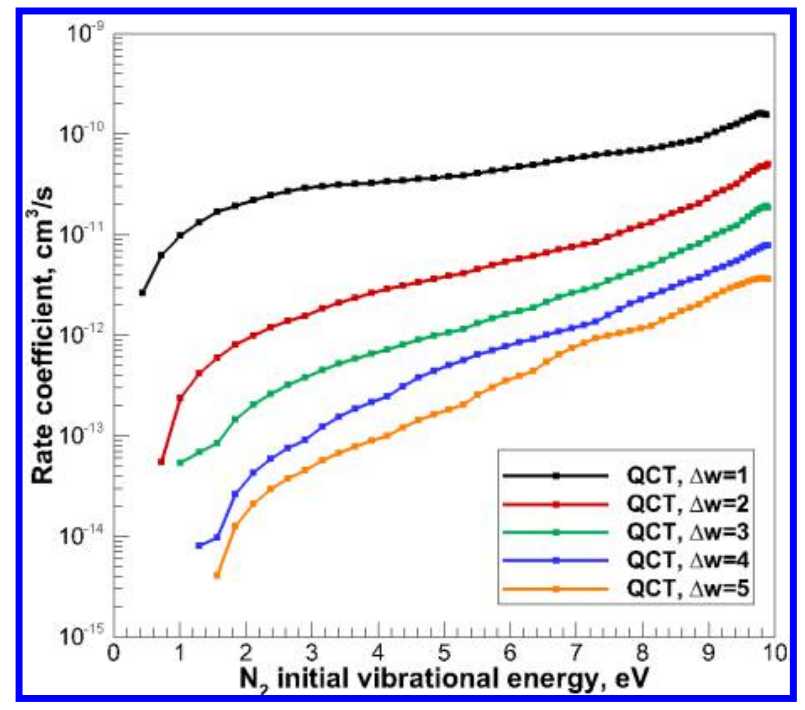

(c) QCT, $\mathrm{O}_{2}(v=20)$

Fig. 6: VT energy transfer RCs for $\mathrm{N}_{2}$ 
The QCT RCs of $\mathrm{O}_{2}$ mono- and multi-quantum transitions in VT collisions with molecular nitrogen are shown in Figs. 7a - 9. The initial vibrational state of nitrogen is set to $w=0$ in Fig. 7a, to $w=5$ in Fig. 7b, to $w=10$ in Fig. $8 \mathrm{a}$, to $\mathrm{w}=20$ in Fig. $8 \mathrm{~b}$ and to $\mathrm{w}=30$ in Fig. 9. For reference, the FHO [47] RCs at T=10,000 $\mathrm{K}$ are shown in Fig. 6a with dashed lines.

As in the case with nitrogen vibrational deactivation by $\mathrm{O}_{2}$, the FHO RCs demonstrate similarity when compared to the QCT data. However, the difference between the FHO and QCT rates is larger in this case. For monoquantum deactivation RCs in collisions with $\mathrm{N}_{2}(w=0)$ satisfactory agreement is achieved for low-lying $\mathrm{O}_{2}$ states. Again, the QCT RCs for excited states of oxygen are higher than those of the FHO model. The disagreement between the FHO and QCT data for multi-quantum is substantially larger than in the case with $\mathrm{N}_{2}$ deactivation (Figs. 6a - 6c). Namely, the FHO model noticeably overestimates the QCT data for almost the entire $\mathrm{O}_{2}$ vibrational ladder. The VT RCs of oxygen vibrational transition demonstrate a little variation with respect to the $\mathrm{N}_{2}$ vibrational quantum state as well.

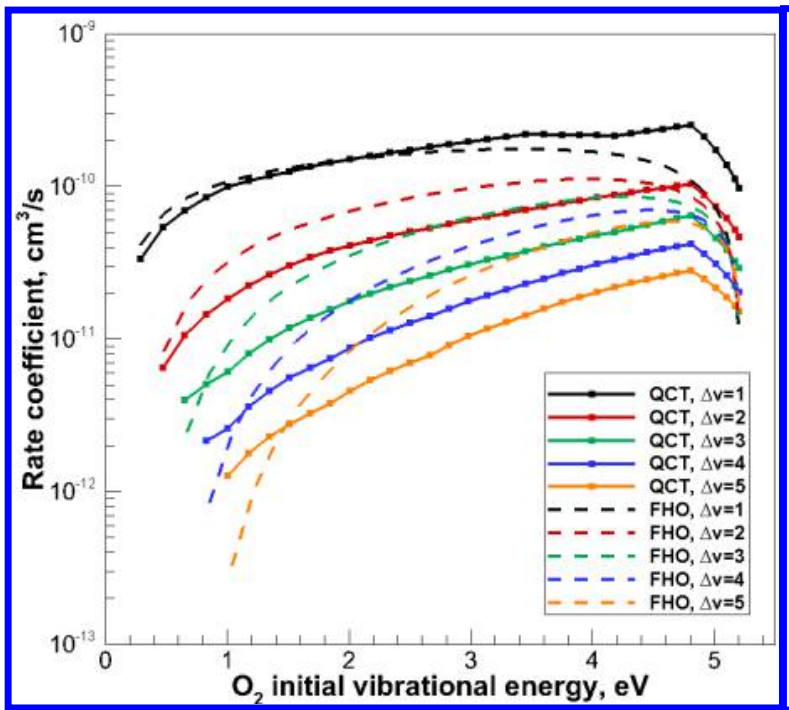

(a) QCT with $\mathrm{N}_{2}(w=0)$ and FHO with $\mathrm{T}=10,000 \mathrm{~K}$

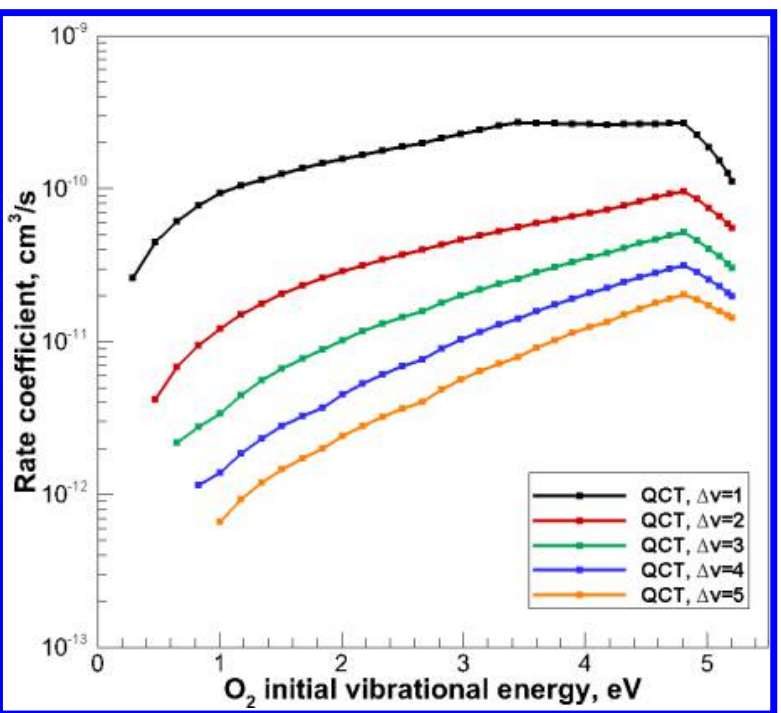

(b) QCT, $\mathrm{N}_{2}(w=10)$

Fig. 7: VT energy transfer RCs for $\mathrm{O}_{2}$

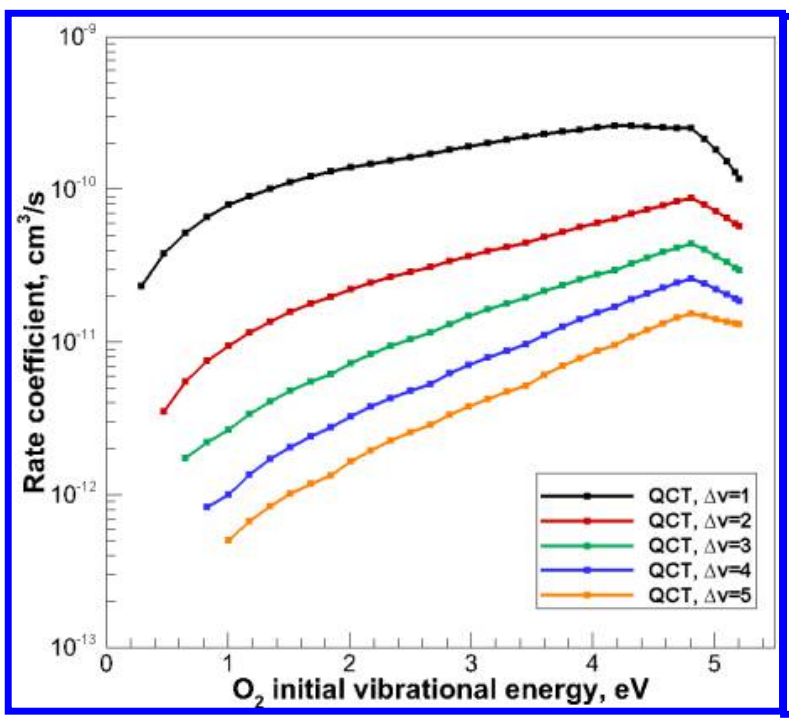

(a) QCT, $\mathrm{N}_{2}(w=20)$

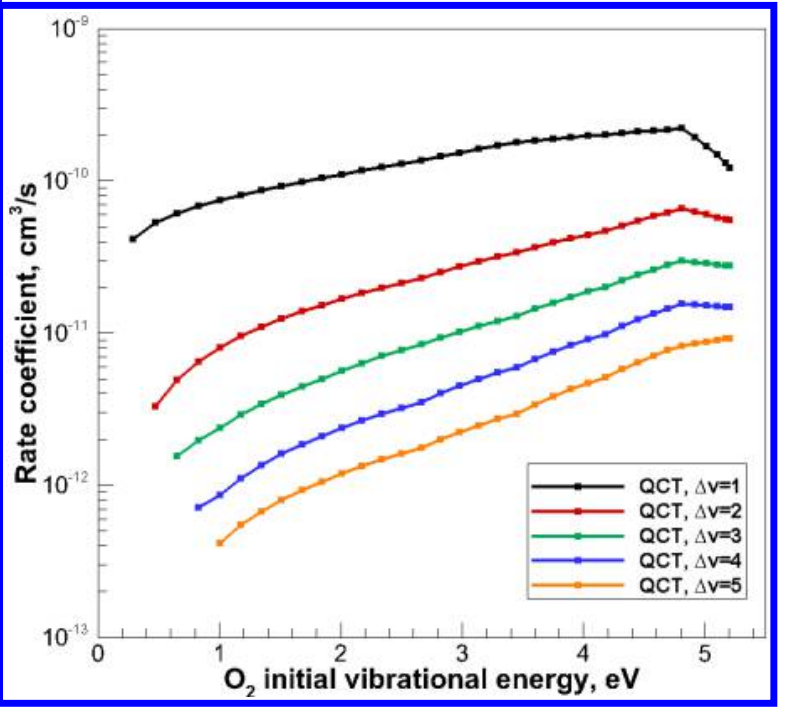

(b) QCT, $\mathrm{N}_{2}(w=30)$

Fig. 8: VT energy transfer RCs for $\mathrm{O}_{2}$ 


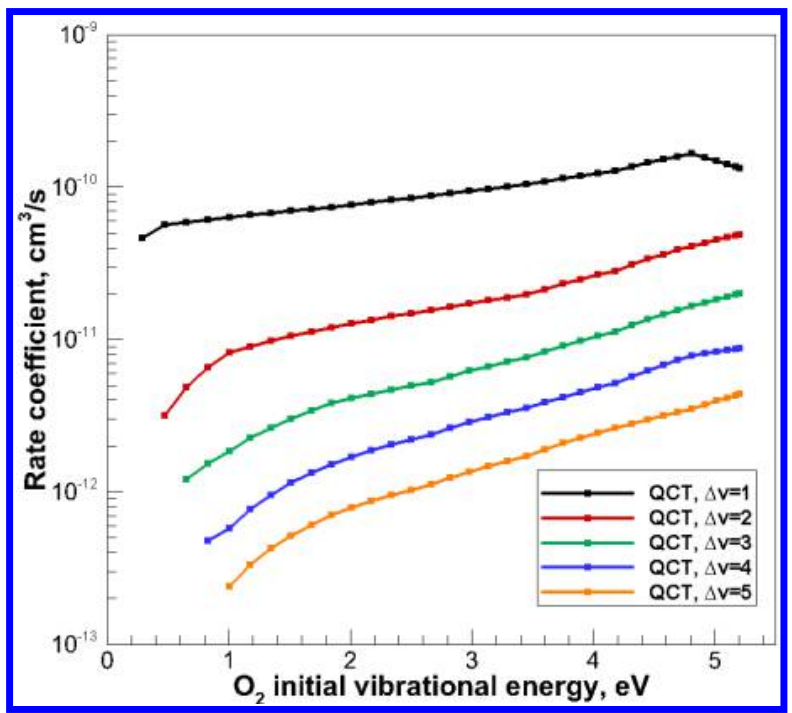

Fig. 9: QCT VT energy transfer RCs for $\mathrm{O}_{2}$ in collisions with $\mathrm{N}_{2}(w=40)$

The rate coefficients for the VV energy transfer mechanism are shown in Figs. 10a - 10b. The abscissa indicates the difference in vibrational energy between products and reactants. In these results, the monoquantum activation of nitrogen is considered, while the oxygen molecules undergo a mono-, double-quantum deactivation. The initial state of nitrogen is set to $w=0$ in Fig. 10a and to $w=10$ in Fig. 10b. For each curve, the initial vibrational state of oxygen increases from left to right.

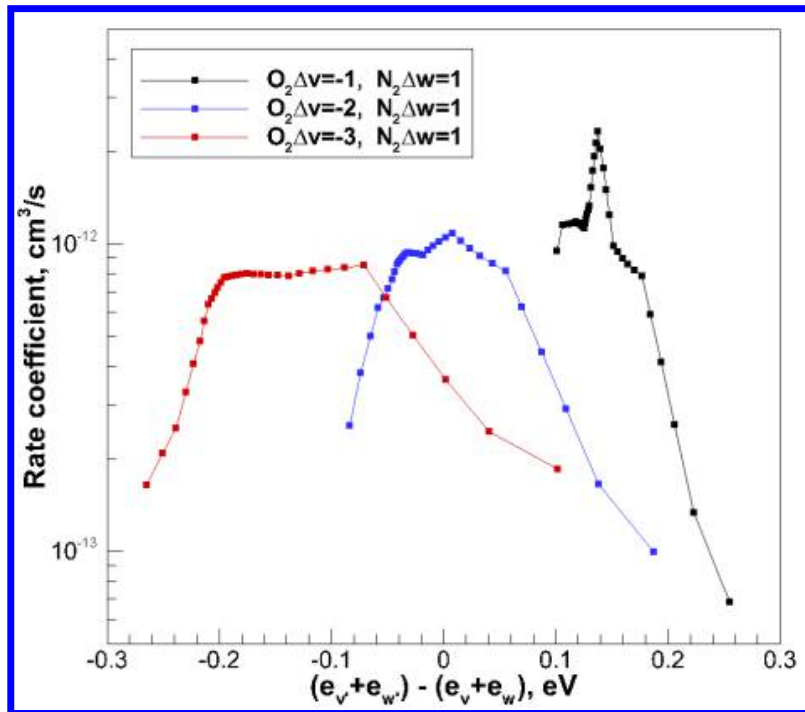

(a) $\mathrm{O}_{2}(v)+\mathrm{N}_{2}(w=0)$

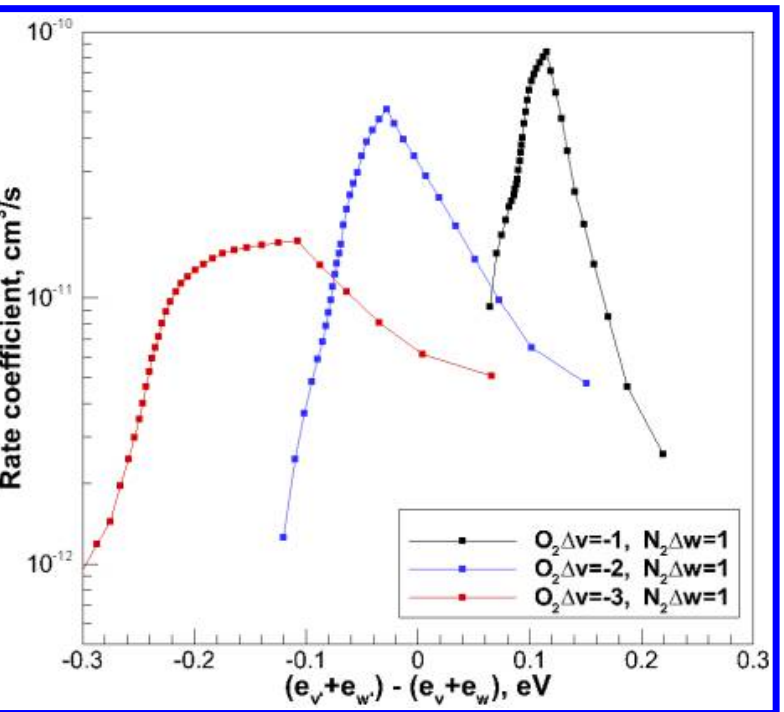

(b) $\mathrm{O}_{2}(v)+\mathrm{N}_{2}(w=10)$

Fig. 10: Rate coefficients of VV energy transfer

Lastly, the interpolated state-specific dissociation rate coefficients are shown in Fig. 11a for $\mathrm{O}_{2}$ and in Fig. 11a for $\mathrm{N}_{2}$. In each of these figures, the abscissa corresponds to the initial vibrational energy of the dissociated reactant, and each curve corresponds to the same initial state of the projectile. As expected, at $\mathrm{T}=10,000 \mathrm{~K}$ molecular oxygen dissociates noticeably faster than nitrogen. The rate of dissociation decreases by almost an order of magnitude in collisions with a vibrationally excited projectile. This means that the internal energy, required to dissociate the target molecule is taken primarily from the translational mode, not from the vibrational mode of the projectile. The RCs for excited vibrational states of the target molecule deviate from a linear dependence on a semi-log scale. This was observed previously for other types of molecular collisions [19]. A similar situation occurs for the state-specific dissociation RCs of nitrogen, as can 
be seen in Fig. 11b. There is some statistical uncertainty associated with the dissociation RCs of low-lying $\mathrm{N}_{2}$ vibrational states. This numerical artifact can be eliminated by increasing the number of trajectories in the QCT simulation. At given equilibrium conditions, molecular oxygen will be almost entirely depleted while nitrogen dissociates insignificantly. Taking into account this fact, the dissociation of $\mathrm{N}_{2}$ has been left for future investigation, and only $\mathrm{O}_{2}$ depletion is implemented in the system of master equations.

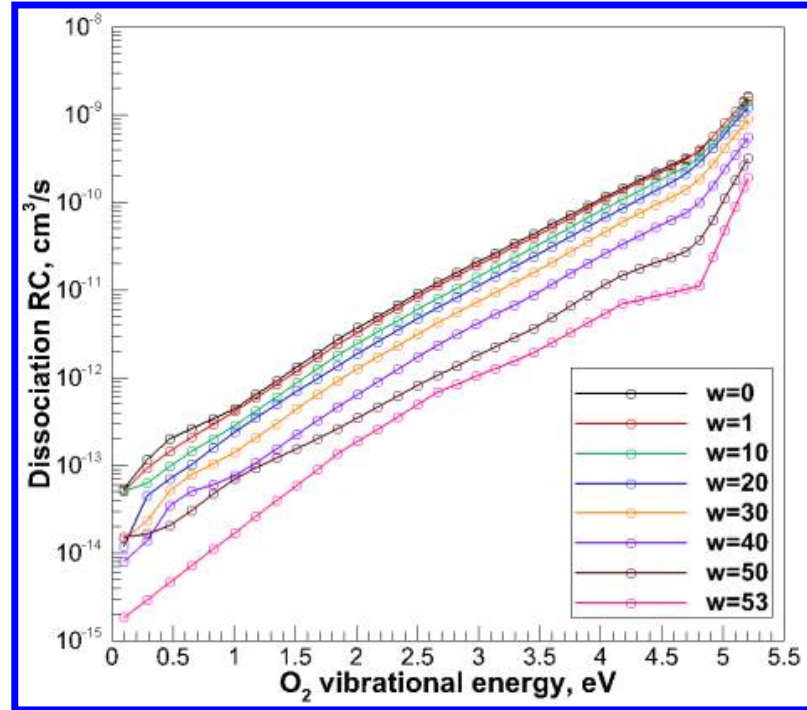

(a) $\mathrm{O}_{2}$

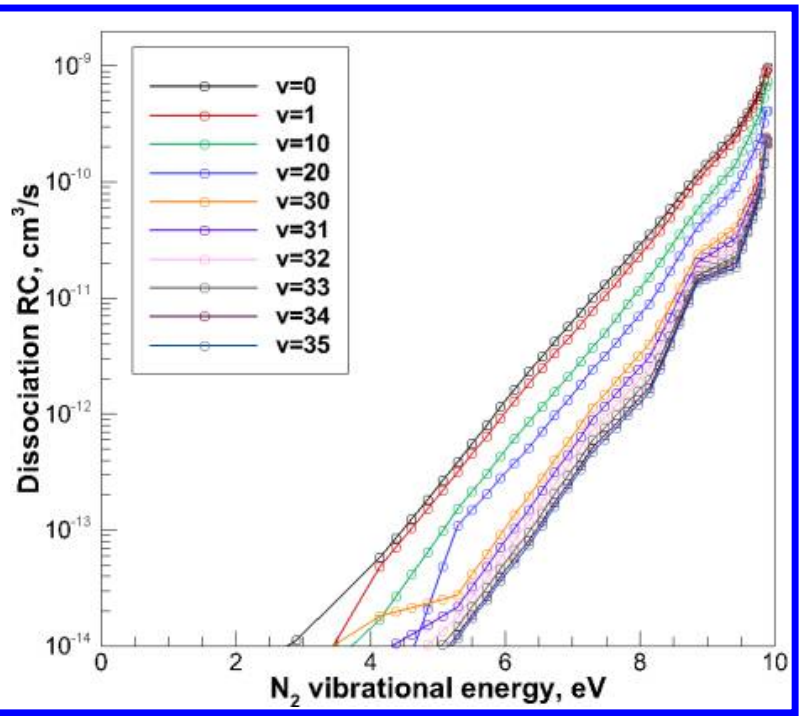

(b) $\mathrm{N}_{2}$

Fig. 11: State-specific dissociation rate coefficients

\section{Master equation simulation}

As discussed previously, there is some ambiguity in specifying initial conditions for a mixture of two molecular species. An initial vibrational temperature of $100 \mathrm{~K}$ is set for both $\mathrm{O}_{2}$ and $\mathrm{N}_{2}$ species, unless otherwise specified. The investigation is conducted in a manner when only VT collisions are included at first. After that, the VV energy transfer mechanism is added to the kinetic model. Finally, the dissociation of oxygen is enabled. In these simulations, the state-specific $\mathrm{N}_{2}$ dissociation RCs are set to zero. Initial total number density is set to $5 \times 10^{18} \mathrm{~cm}^{-3}$ with the molar ratio of oxygen and nitrogen of 1:4.

The variation of $\mathrm{O}_{2}$ and $\mathrm{N}_{2}$ vibrational temperature when only VT collisions are taken into account is shown in Fig. 12. Black curves correspond to the situation when only transitions with $|\Delta v|=1$ and $|\Delta w|=1$ are allowed. Blue, red, green and gray curves describe relaxation with multiquantum jumps, respectively. The purpose of such a comparison is to demonstrate the influence of multi-quantum jumps on the relaxation process. Multi-quantum jumps make oxygen vibrational relaxation significantly faster, while the multi-quantum relaxation mechanisms are of secondary importance for nitrogen. This is, obviously, explained by the large difference in the size of vibrational quanta for these two species. Overall, multiquantum mechanisms decrease $\mathrm{O}_{2}$ relaxation time by a factor of 2.8 .

The system of master equations resolves the temporal evolution of state-specific populations during thermalization process. Actual (nonequilibrium) and Boltzmann populations, estimated from the vibrational temperature of species, are shown in Figs. 13a and 13b for $\mathrm{N}_{2}$ and $\mathrm{O}_{2}$, respectively.

The variation of $\mathrm{O}_{2}$ and $\mathrm{N}_{2}$ vibrational temperatures with time is shown in Fig. 14. Three sets represent the calculations when the energy is transfered only in VT collisions (solid line), in VT and VV transitions with $|\Delta v| \leq 1$ and $|\Delta w| \leq 1$ (short dashed line) and in VT and VV transitions with $|\Delta v| \leq 2$ and $|\Delta w| \leq 2$ (long dashed line). This numerical experiment demonstrates the influence of the VV energy transfer on vibrational temperature at hypersonic temperatures.

As expected, the relaxation of oxygen in collisions with $\mathrm{N}_{2}$ occurs faster, than the relaxation of nitrogen. When the VV energy transfer mechanism with single quantum jumps is implemented, the relaxation of oxygen becomes slower at the late stage of relaxation, compared to the case when only VT transitions are considered. At the same time, the nitrogen vibrational relaxation time becomes shorter. This indicates that 


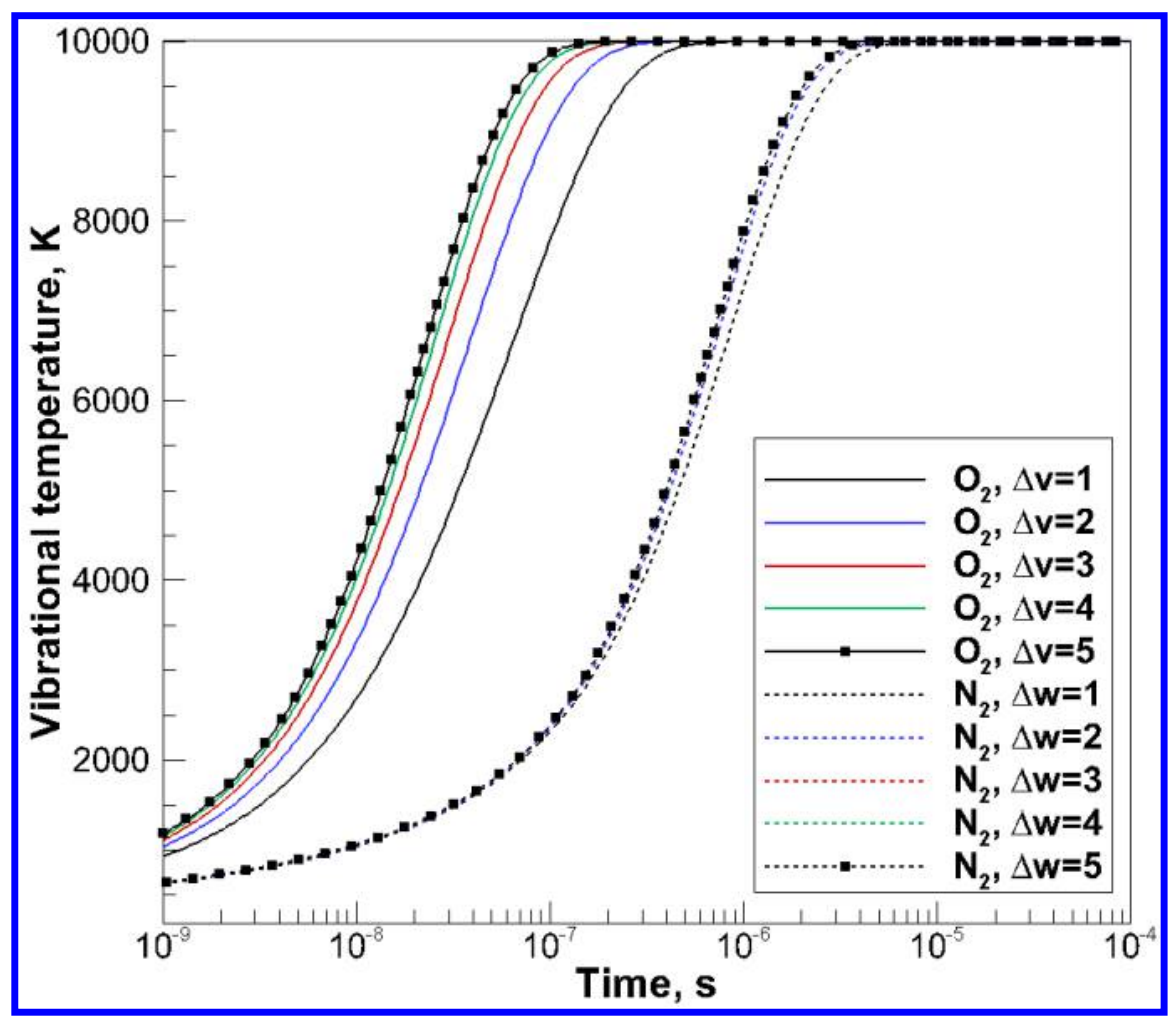

Fig. 12: Variation of vibrational temperature, only VT collisions are taken into account

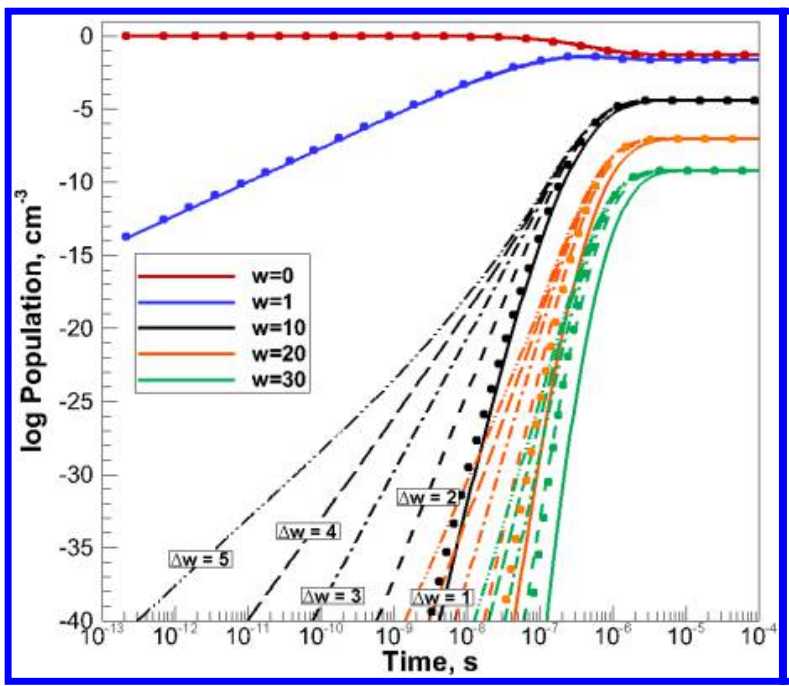

(a) Nitrogen vibrational ladder

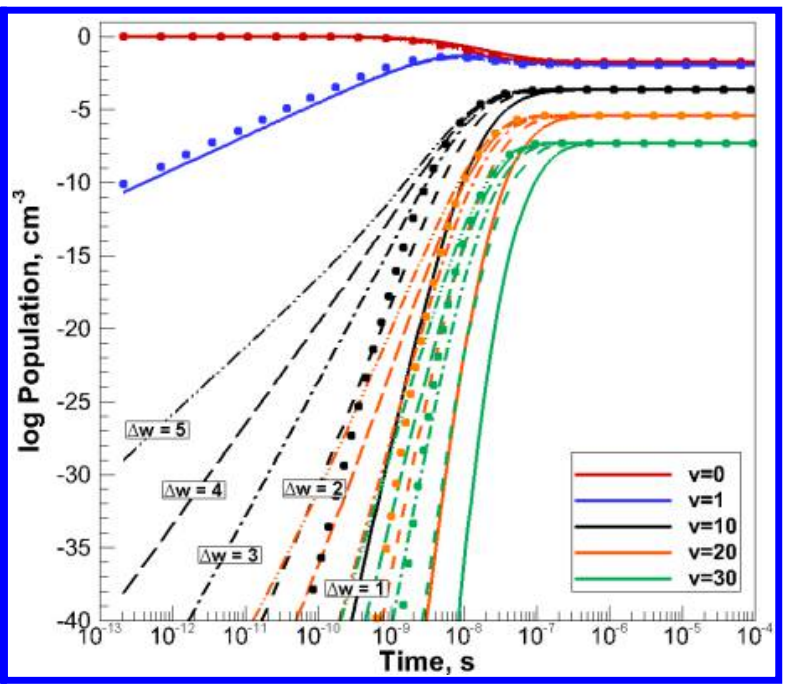

(b) Oxygen vibrational ladder

Fig. 13: Population of vibrational ladder, $T=T_{r}=10,000 \mathrm{~K}$. Only VT collisions are simulated. 
the energy is transfered from oxygen vibrational states to the nitrogen vibrational ladder. This direction of energy transfer is in the agreement with the assumptions made in the work by White [41]. The double quantum VV transitions bring minor changes in the relaxation time of considered species. A summary of relaxation times, obtained for both $\mathrm{O}_{2}$ and $\mathrm{N}_{2}$ species, is provided in Table 3 .

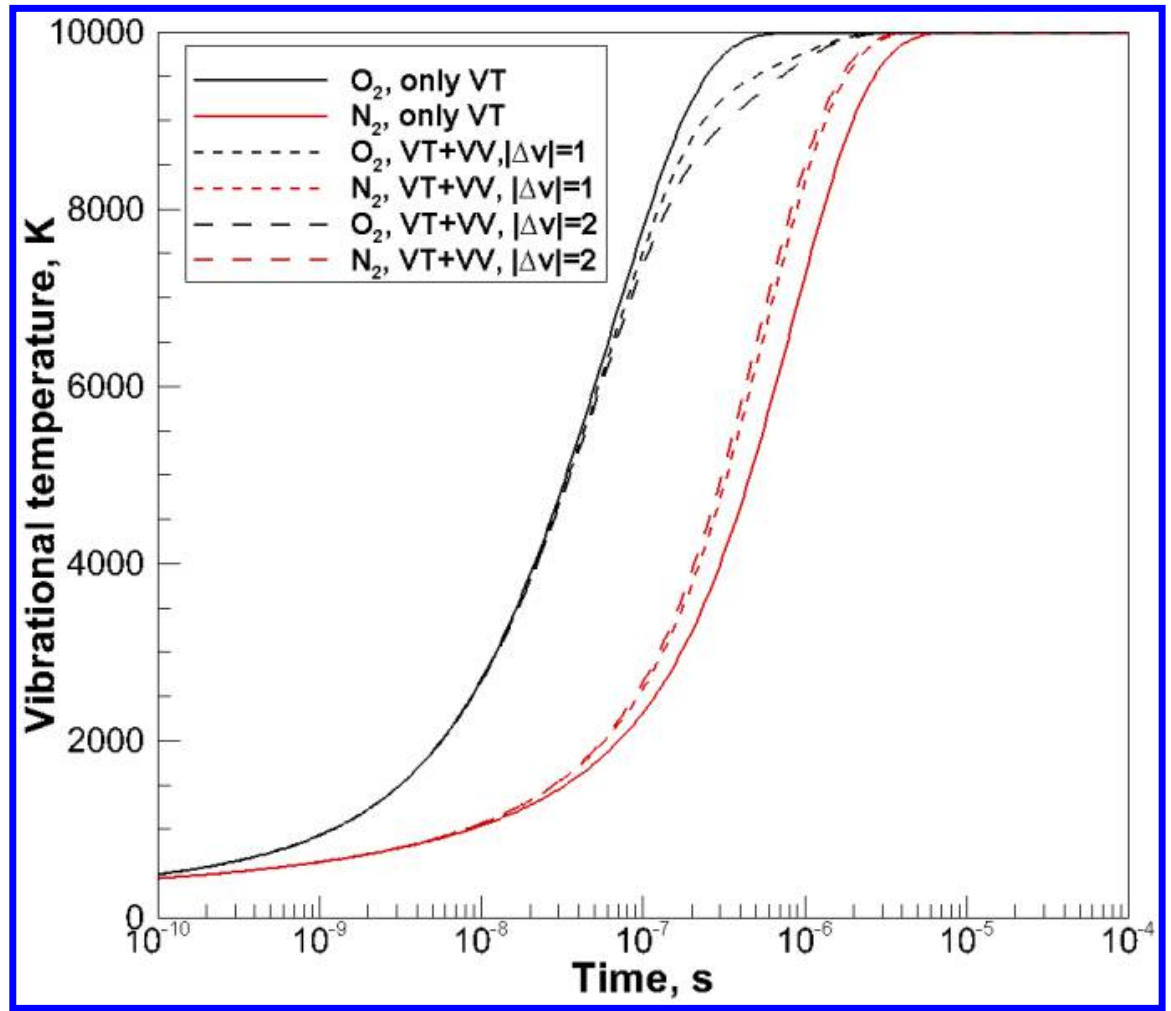

Fig. 14: $\mathrm{O}_{2}$ and $\mathrm{N}_{2}$ vibrational temperature obtained via master equation simulation using sets of VT and VV transitions

The present paper also features the thermal relaxation of $\mathrm{O}_{2}$ and $\mathrm{N}_{2}$ species in the presence of dissociation. At an equilibrium temperature of $10000 \mathrm{~K}$, the depletion of oxygen is nearly complete while nitrogen dissociates much slower and only partially. For this reason, an implementation of $\mathrm{N}_{2}$ dissociation RCs is left for the future, and only $\mathrm{O}_{2}$ dissociation is considered with the state-specific RCs shown in Fig. 11a.

The variation of vibrational temperature with time for both $\mathrm{O}_{2}$ and $\mathrm{N}_{2}$ species is shown in Fig. 15. In these calculations, the initial vibrational temperature is set to $100 \mathrm{~K}$ and the overall number density of particles is $5 \times 10^{18} \mathrm{~cm}^{-3}$. Unlike in molecule-atom master equation simulations [19,48], the vibrational temperature of oxygen flattens out twice during thermalization to equilibrium conditions. Clearly one plateau corresponds to the "classical" quasi-steady state, when active dissociation occurs from highly excited vibrational states while the $\mathrm{O}_{2}$ vibrational energy remains nearly constant. The second plateau corresponds to the vibrational relaxation of nitrogen. At this moment, the transfer of vibrational energy from oxygen to nitrogen occurs, as shown in Fig. 14. The evolution of $\mathrm{O}_{2}$ number density is shown in Fig. 16. Along with this data, the instantaneous and equilibrium dissociation RCs are shown with solid and dashed black curves. The atomic oxygen number density reaches 0.15 of its equilibrium value by the beginning of the QSS state. This indicates that the ubiquitous assumption about separation of the QSS state and vibrational relaxation may not be accurate at the given heat bath conditions. The equilibrium dissociation RC is approximately 7 times larger than the instantaneous dissociation RCs, which is explained by an incomplete thermalization of the $\mathrm{O}_{2}$ vibrational ladder.

\section{Conclusion}

Vibrational energy transfer in $\mathrm{O}_{2}-\mathrm{N}_{2}$ collisions is studied by means of the QCT method and a system of master equations. The rate coefficients of vibrational-translation and vibration-vibration energy transfer are 


\begin{tabular}{|c|c|c|c|}
\hline Target molecule & Initial state & Relax. time, s & Note \\
\hline $\mathrm{O}_{2}$ & $T_{v}\left(\mathrm{O}_{2}\right)=T_{v}\left(\mathrm{~N}_{2}\right)=100 \mathrm{~K}$ & $3.559 \times 10^{-7}$ & only VT transitions $\Delta v \leq 1, \Delta w \leq 1$ \\
\hline $\mathrm{O}_{2}$ & $T_{v}\left(\mathrm{O}_{2}\right)=T_{v}\left(\mathrm{~N}_{2}\right)=100 \mathrm{~K}$ & $2.127 \times 10^{-7}$ & only VT transitions $\Delta v \leq 2, \Delta w \leq 2$ \\
\hline $\mathrm{O}_{2}$ & $T_{v}\left(\mathrm{O}_{2}\right)=T_{v}\left(\mathrm{~N}_{2}\right)=100 \mathrm{~K}$ & $1.622 \times 10^{-7}$ & only VT transitions $\Delta v \leq 3, \Delta w \leq 3$ \\
\hline $\mathrm{O}_{2}$ & $T_{v}\left(\mathrm{O}_{2}\right)=T_{v}\left(\mathrm{~N}_{2}\right)=100 \mathrm{~K}$ & $1.388 \times 10^{-7}$ & only VT transitions $\Delta v \leq 4, \Delta w \leq 4$ \\
\hline $\mathrm{O}_{2}$ & $T_{v}\left(\mathrm{O}_{2}\right)=T_{v}\left(\mathrm{~N}_{2}\right)=100 \mathrm{~K}$ & $1.264 \times 10^{-7}$ & only VT transitions $\Delta v \leq 5, \Delta w \leq 5$ \\
\hline $\mathrm{N}_{2}$ & $T_{v}\left(\mathrm{O}_{2}\right)=T_{v}\left(\mathrm{~N}_{2}\right)=100 \mathrm{~K}$ & $1.267 \times 10^{-6}$ & only VT transitions $\Delta v \leq 1, \Delta w \leq 1$ \\
\hline $\mathrm{N}_{2}$ & $T_{v}\left(\mathrm{O}_{2}\right)=T_{v}\left(\mathrm{~N}_{2}\right)=100 \mathrm{~K}$ & $1.098 \times 10^{-6}$ & only VT transitions $\Delta v \leq 2, \Delta w \leq 2$ \\
\hline $\mathrm{N}_{2}$ & $T_{v}\left(\mathrm{O}_{2}\right)=T_{v}\left(\mathrm{~N}_{2}\right)=100 \mathrm{~K}$ & $1.043 \times 10^{-6}$ & only VT transitions $\Delta v \leq 3, \Delta w \leq 3$ \\
\hline $\mathrm{N}_{2}$ & $T_{v}\left(\mathrm{O}_{2}\right)=T_{v}\left(\mathrm{~N}_{2}\right)=100 \mathrm{~K}$ & $1.025 \times 10^{-6}$ & only VT transitions $\Delta v \leq 4, \Delta w \leq 4$ \\
\hline $\mathrm{N}_{2}$ & $T_{v}\left(\mathrm{O}_{2}\right)=T_{v}\left(\mathrm{~N}_{2}\right)=100 \mathrm{~K}$ & $1.017 \times 10^{-6}$ & only VT transitions $\Delta v \leq 5, \Delta w \leq 5$ \\
\hline $\mathrm{O}_{2}$ & $T_{v}\left(\mathrm{O}_{2}\right)=T_{v}\left(\mathrm{~N}_{2}\right)=100 \mathrm{~K}$ & $1.293 \times 10^{-7}$ & $\begin{aligned} \operatorname{VT}(\Delta v \leq 5, \Delta w \leq 5) \\
\operatorname{VV}(\Delta v \leq 1, \Delta w \leq 1)\end{aligned}$ \\
\hline $\mathrm{O}_{2}$ & $T_{v}\left(\mathrm{O}_{2}\right)=T_{v}\left(\mathrm{~N}_{2}\right)=100 \mathrm{~K}$ & $1.312 \times 10^{-7}$ & $\begin{array}{l}\mathrm{VT}(\Delta v \leq 5, \Delta w \leq 5) \\
\mathrm{VV}(\Delta v \leq 2, \Delta w \leq 2)\end{array}$ \\
\hline $\mathrm{O}_{2}$ & $T_{v}\left(\mathrm{O}_{2}\right)=T_{v}\left(\mathrm{~N}_{2}\right)=100 \mathrm{~K}$ & $1.324 \times 10^{-7}$ & $\begin{aligned} \mathrm{VT} & (\Delta v \leq 5, \Delta w \leq 5) \\
\operatorname{VV}(\Delta v & \leq 3, \Delta w \leq 3)\end{aligned}$ \\
\hline $\mathrm{O}_{2}$ & $T_{v}\left(\mathrm{O}_{2}\right)=T_{v}\left(\mathrm{~N}_{2}\right)=100 \mathrm{~K}$ & $1.331 \times 10^{-7}$ & $\begin{aligned} \mathrm{VT}(\Delta v & \leq 5, \Delta w \leq 5) \\
\mathrm{VV}(\Delta v & \leq 4, \Delta w \leq 4)\end{aligned}$ \\
\hline $\mathrm{N}_{2}$ & $T_{v}\left(\mathrm{O}_{2}\right)=T_{v}\left(\mathrm{~N}_{2}\right)=100 \mathrm{~K}$ & $7.430 \times 10^{-7}$ & $\begin{aligned} \mathrm{VT}(\Delta v & \leq 5, \Delta w \leq 5) \\
\mathrm{VV}(\Delta v & \leq 1, \Delta w \leq 1)\end{aligned}$ \\
\hline $\mathrm{N}_{2}$ & $T_{v}\left(\mathrm{O}_{2}\right)=T_{v}\left(\mathrm{~N}_{2}\right)=100 \mathrm{~K}$ & $6.523 \times 10^{-7}$ & $\begin{aligned} & \mathrm{VT}(\Delta v \leq 5, \Delta w \leq 5) \\
& \operatorname{VV}(\Delta v \leq 2, \Delta w \leq 2)\end{aligned}$ \\
\hline $\mathrm{N}_{2}$ & $T_{v}\left(\mathrm{O}_{2}\right)=T_{v}\left(\mathrm{~N}_{2}\right)=100 \mathrm{~K}$ & $6.169 \times 10^{-7}$ & $\begin{array}{c}\mathrm{VT}(\Delta v \leq 5, \Delta w \leq 5) \\
\mathrm{VV}(\Delta v \leq 3, \Delta w \leq 3)\end{array}$ \\
\hline $\mathrm{N}_{2}$ & $T_{v}\left(\mathrm{O}_{2}\right)=T_{v}\left(\mathrm{~N}_{2}\right)=100 \mathrm{~K}$ & $6.021 \times 10^{-7}$ & $\begin{aligned} \mathrm{VT}(\Delta v & \leq 5, \Delta w \leq 5) \\
\mathrm{VV}(\Delta v & \leq 4, \Delta w \leq 4)\end{aligned}$ \\
\hline $\mathrm{O}_{2}$ & $T_{v}\left(\mathrm{O}_{2}\right)=T_{v}\left(\mathrm{~N}_{2}\right)=100 \mathrm{~K}$ & $1.258 \times 10^{-7}$ & only VT transitions of oxygen $\Delta v \leq 5$ \\
\hline $\mathrm{O}_{2}$ & $\begin{array}{c}T_{v}\left(\mathrm{O}_{2}\right)=100 \mathrm{~K} \\
T_{v}\left(\mathrm{~N}_{2}\right)=10,000 \mathrm{~K}\end{array}$ & $1.492 \times 10^{-7}$ & only VT transitions of oxygen $\Delta v \leq 5$ \\
\hline
\end{tabular}

Table 3: Vibrational relaxation times, derived from master equation simulations

generated at hypersonic temperatures in the range between 1000 and $10,000 \mathrm{~K}$ as well as at room temperatures. The latter was performed in order to compare the results of the QCT simulation with experimental data obtained for vibrationally excited oxygen.

At room temperature, the present ab-initio PES gives significantly higher transitions rates compared to the experimental data by Park and Slanger. One possible explanation for this is the influence of the long-distance forces, implemented in Varga's PES, which appears to be more attractive, when compared to the PES developed by Billing. An overestimation of RCs is also observed when the present results are compared to the RCs by Billing, obtained via the semiclassical trajectory method on a different PES. The QCT method can be another source of inaccuracy. Since the classical propagation of trajectory does not take into account some important non-classical phenomena, it is recommended to revisit these calculations using more rigorous trajectory propagation methods.

Nevertheless, the implemented PES demonstrated good accuracy of rate coefficients at high collision energies, as can be judged by comparing the QCT data with the shock tube measurements. Indeed, when collisions occur with high translational energy, the attractive forces of the PES become relatively unimportant compared to the repulsive wall of the potential. The vibrational relaxation time of oxygen in collisions with 


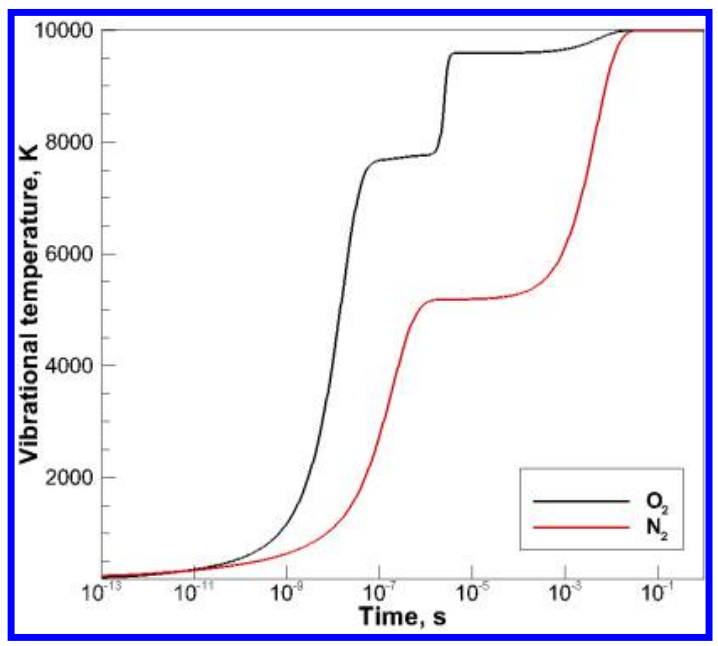

Fig. 15: Vibrational temperature of oxygen and nitrogen, $\mathrm{O}_{2}$ dissociation is considered at heat bath of $10,000 \mathrm{~K}$

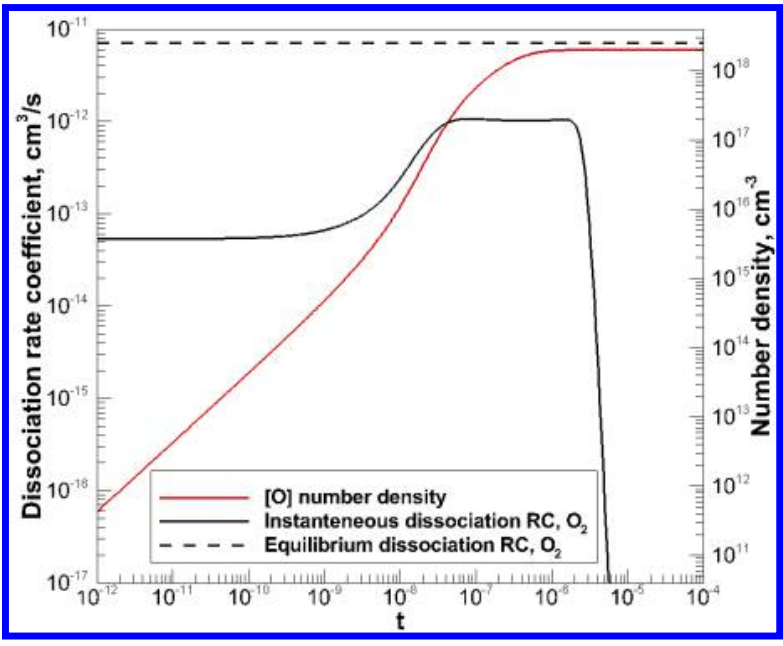

Fig. 16: Instantaneous and equilibrium $\mathrm{O}_{2}$ dissociation RCs and atomic oxygen number density at heat bath of $10,000 \mathrm{~K}$

$\mathrm{N}_{2}$ is well described by the experimental data by Losev and General in the temperature range between 4000 and $6000 \mathrm{~K}$. The difficulties and inaccuracies of the QCT approach are recognized when attempting to generate the state-specific RCs for temperatures below $4000 \mathrm{~K}$. This can be attributed to the failure of the QCT method to describe quantum effects at low temperatures and for low-lying vibrational states. At these temperatures, the QCT method strongly underestimates the true RCs, that can be obtained by a more rigorous approach at higher computational cost. Since these temperatures are still of interest for hypersonic simulations, a combination of semi-classical and quasi-classical trajectory approaches is recommended for future investigation in the field of state-resolved chemical kinetics. Meanwhile, the thermal equilibrium dissociation RC is in agreement with the ARAS measurements by Jerig et al. [46] at temperatures between 2000 and $3700 \mathrm{~K}$. At higher temperatures, the present dissociation RC agrees well with the data obtained by measuring the laser absorption in the Schumann-Runge bands [44].

A set of vibrationally resolved $\mathrm{O}_{2}-\mathrm{N}_{2}$ rate coefficients is generated at $10,000 \mathrm{~K}$, in order to model thermal relaxation of both species using master equations. The rate coefficients are generated for 160 combinations of vibrational levels by the QCT method. The rest of the RCs are interpolated. The solution of master equations indicated that multiquantum transitions are crucially important for oxygen relaxation, specifically for kinetics of excited vibrational states. The effect of multi-quantum jumps on nitrogen relaxation is less pronounced. The vibration-vibration collisions transfer energy from oxygen to the nitrogen vibrational ladder, extending the oxygen relaxation time. This effect is quite significant and, in fact, has an impact on the vibrational temperature of the species.

\section{Acknowledgment}

The authors gratefully acknowledge funding for this work through Air Force Office of Scientific Research Grant FA9550-16-1-0291.

\section{References}

${ }^{1}$ A. Varandas and A. Pais, "Double many-body expansion potential energy surface for $\mathrm{O}_{4}\left({ }^{3} \mathrm{~A}\right)$, dynamics of the $\mathrm{O}\left({ }^{3} \mathrm{P}\right)+$ $\mathrm{O}_{3}\left({ }^{1} \mathrm{~A}_{1}\right)$ reaction, and second virial coefficients of molecular oxygen," in Theoretical and Computational Models for Organic Chemistry, pp. 55-78, Springer, 1991.

${ }^{2}$ V. Aquilanti, M. Bartolomei, E. Carmona-Novillo, and F. Pirani, "The asymmetric dimer $\mathrm{N}_{2}-\mathrm{O}_{2}$ : characterization of the potential energy surface and quantum mechanical calculation of rotovibrational levels," The Journal of chemical physics, vol. 118, no. 5, pp. 2214-2222, 2003.

${ }^{3}$ R. Schinke, S. Y. Grebenshchikov, M. Ivanov, and P. Fleurat-Lessard, "Dynamical studies of the ozone isotope effect: A status report," Annu. Rev. Phys. Chem., vol. 57, pp. 625-661, 2006.

$>^{4}$ J. D. Bender, P. Valentini, I. Nompelis, T. Schwartzentruber, and G. V. Candler, "Characterization of vibrational and ro- 
tational energy transfer in $\mathrm{N}_{2}+\mathrm{N}_{2}$ dissociative collisions using the quasiclassical trajectory method," 45th AIAA Thermophysics Conference, AIAA paper 2015-3253, June 2015.

${ }^{5}$ M. Panesi, R. L. Jaffe, D. W. Schwenke, and T. E. Magin, "Rovibrational internal energy transfer and dissociation of $\mathrm{N}_{2}\left({ }^{1} \Sigma_{g}^{+}\right)-\mathrm{N}\left({ }^{4} \mathrm{~S}_{u}\right)$ system in hypersonic flows," Journal of Chemical Physics, vol. 138, no. 4, p. 044312, 2013.

${ }^{6}$ D. Bruno, M. Capitelli, F. Esposito, S. Longo, and P. Minelli, "Direct simulation of non-equilibrium kinetics under shock conditions in nitrogen," Chemical physics letters, vol. 360, no. 1, pp. 31-37, 2002.

${ }^{7}$ F. Esposito, M. Capitelli, and C. Gorse, "Quasi-classical dynamics and vibrational kinetics of $\mathrm{N}+\mathrm{N}_{2}(v)$ system," Chemical Physics, vol. 257, no. 2, pp. 193-202, 2000.

8 J. G. Kim and I. D. Boyd, "State-resolved master equation analysis of thermochemical nonequilibrium of nitrogen," Chemical Physics, vol. 415, pp. 237-246, 2013.

${ }^{9}$ D. Bose, G. V. Candler, et al., "Thermal rate constants of the $\mathrm{O}_{2}+\mathrm{N} \rightarrow \mathrm{NO}+\mathrm{O}$ reaction based on the ${ }^{2} A^{\prime}$ and ${ }^{4} A^{\prime}$ potential-energy surfaces," Journal of Chemical Physics, vol. 107, no. 16, pp. 6136-6145, 1997.

${ }^{10} \mathrm{~J}$. W. Duff and R. D. Sharma, "Quasiclassical trajectory study of NO vibrational relaxation by collisions with atomic oxygen," Journal of the Chemical Society, Faraday Transactions, vol. 93, no. 16, pp. 2645-2649, 1997.

${ }^{11}$ I. D. Boyd, D. Bose, and G. V. Candler, "Monte carlo modeling of nitric oxide formation based on quasi-classical trajectory calculations," Physics of Fluids (1994-present), vol. 9, no. 4, pp. 1162-1170, 1997.

${ }^{12} \mathrm{M}$. González, R. Valero, and R. Sayós, "Ab initio and quasiclassical trajectory study of the $\mathrm{n}(2 \mathrm{~d})+\mathrm{no}(\mathrm{x} 2 \pi) \mathrm{o}(1 \mathrm{~d})+\mathrm{n} 2(\mathrm{x}$ $1 \sigma \mathrm{g}+$ ) reaction on the lowest 1a potential energy surface," The Journal of Chemical Physics, vol. 113, no. 24, pp. 10983-10998, 2000.

${ }^{13}$ J. C. Castro-Palacio, T. Nagy, R. J. Bemish, and M. Meuwly, "Computational study of collisions between $\mathrm{O}\left({ }^{3} \mathrm{P}\right)$ and NO $\left({ }^{2} \Pi\right)$ at temperatures relevant to the hypersonic flight regime," The Journal of chemical physics, vol. 141, no. 16, p. 164319, 2014.

${ }^{14}$ K. Yamashita, K. Morokuma, F. Le Quéré, and C. Leforestier, "New ab initio potential surfaces and three-dimensional quantum dynamics for transition state spectroscopy in ozone photodissociation," Chemical Physics Letters, vol. 191, no. 6, pp. 515-520, 1992.

${ }^{15}$ R. Siebert, P. Fleurat-Lessard, R. Schinke, M. Bittererová, and S. Farantos, "The vibrational energies of ozone up to the dissociation threshold: Dynamics calculations on an accurate potential energy surface," Journal of Chemical Physics, vol. 116, no. 22, p. 9749, 2002.

${ }^{16}$ V. G. Tyuterev, S. Tashkun, P. Jensen, A. Barbe, and T. Cours, "Determination of the effective ground state potential energy function of ozone from high-resolution infrared spectra," Journal of Molecular Spectroscopy, vol. 198, no. 1, pp. 57-76, 1999.

${ }^{17}$ A. Varandas and A. Pais, "A realistic double many-body expansion (DMBE) potential energy surface for ground-state $\mathrm{O}_{3}$ from a multiproperty fit to ab initio calculations, and to experimental spectroscopic, inelastic scattering, and kinetic isotope thermal rate data," Molecular Physics, vol. 65, pp. 843-860, Nov 1988.

${ }^{18} \mathrm{H}$. Webster III and E. J. Bair, "Ozone ultraviolet photolysis. IV. $\mathrm{O}_{2}+\mathrm{O}\left({ }^{3} \mathrm{P}\right)$ vibrational energy transfer," Journal of Chemical Physics, vol. 56, pp. 6104-6108, 1972.

${ }^{19}$ D. A. Andrienko and I. D. Boyd, "Rovibrational energy transfer and dissociation in $\mathrm{O}_{2}-\mathrm{O}$ collisions," The Journal of Chemical Physics, vol. 144, no. 10, p. 104301, 2016.

${ }^{20}$ F. Esposito, I. Armenise, G. Capitta, and M. Capitelli, "O $+\mathrm{O}_{2}$ state-to-state vibrational relaxation and dissociation rates based on quasiclassical calculations," Chemical Physics, vol. 351, no. 1-3, pp. 91-98, 2008.

${ }^{21}$ C. Coletti and G. D. Billing, "Vibrational energy transfer in molecular oxygen collisions," Chemical Physics Letters, vol. 356, no. 1, pp. 14-22, 2002.

${ }^{22}$ G. D. Billing and R. Kolesnick, "Vibrational relaxation of oxygen. state to state rate constants," Chemical Physics Letters, vol. 200, no. 4, pp. 382-386, 1992.

-23 G. D. Billing, "VV and VT rates in $\mathrm{N}_{2}-\mathrm{O}_{2}$ collisions," Chemical Physics, vol. 179, no. 3, pp. 463-467, 1994.

${ }^{24} \mathrm{H}$. Park and T. Slanger, " $\mathrm{O}_{2}(\mathrm{X}, v=8-22) 300 \mathrm{~K}$ quenching rate coefficients for $\mathrm{O}_{2}$ and $\mathrm{N}_{2}$, and $\mathrm{O}_{2}(\mathrm{X})$ vibrational distribution from $248 \mathrm{~nm} \mathrm{O} 3$ photodissociation," The Journal of Chemical Physics, vol. 100, no. 1, pp. 287-300, 1994.

${ }^{25}$ J. Price, J. Mack, C. Rogaski, and A. Wodtke, "Vibrational-state-specific self-relaxation rate constant. measurements of highly vibrationally excited $\mathrm{O}_{2}(\nu=19-28)$," Chemical Physics, vol. 175, no. 1, pp. 83-98, 1993.

${ }^{26}$ A. Varandas, "Are vibrationally excited molecules a clue for the $\mathrm{O}_{3}$ deficit problem and $\mathrm{HO}_{x}$ dilemma in the middle atmosphere?," The Journal of Physical Chemistry A, vol. 108, no. 5, pp. 758-769, 2004.

${ }^{27}$ Y. Paukku, K. R. Yang, Z. Varga, and D. G. Truhlar, "Global ab initio ground-state potential energy surface of $\mathrm{N}_{4}$," The Journal of Chemical Physics, vol. 139, no. 4, p. 044309, 2013.

${ }^{28}$ Z. Varga, R. Meana-Pañeda, G. Song, Y. Paukku, and D. G. Truhlar, "Potential energy surface of triplet $\mathrm{N}_{2} \mathrm{O}_{2}$," The Journal of Chemical Physics, vol. 144, no. 2, p. 024310, 2016.

${ }^{29}$ R. Jaffe, D. Schwenke, and G. Chaban, "Vibrational and rotational excitation and dissociation in $\mathrm{N}_{2}-\mathrm{N}_{2}$ collisions from accurate theoretical calculations," 10th AIAA/ASME Joint Thermophysics and Heat Transfer Conference, AIAA Paper 20104517, June 2010.

${ }^{30}$ K. Koura, "Monte carlo direct simulation of rotational relaxation of diatomic molecules using classical trajectory calculations: Nitrogen shock wave," Physics of Fluids, vol. 9, no. 11, pp. 3543-3549, 1997.

${ }^{31}$ G. D. Billing, "Semiclassical theory for diatom-diatom collisions," Chemical Physics Letters, vol. 97, no. 2, pp. 188-192, 1983.

${ }^{32}$ L. M. Raff, D. L. Thompson, L. Sims, and R. N. Porter, "Dynamics of the molecular and atomic mechanisms for the hydrogen-iodine exchange reaction," The Journal of Chemical Physics, vol. 56, no. 12, pp. 5998-6027, 1972.

${ }^{33}$ D. Andrienko and I. D. Boyd, "Investigation of oxygen vibrational relaxation by quasi-classical trajectory method," Chemical Physics, vol. 459, pp. 1-13, 2015. 
${ }^{34}$ C. Park, "Rotational relaxation of $\mathrm{N}_{2}$ behind a strong shock wave," Journal of Thermophysics and Heat Transfer, vol. 18, no. 4, pp. 527-533, 2004.

${ }^{35}$ M. Quack and J. Troe, "Complex formation in reactive and inelastic scattering: Statistical adiabatic channel model of unimolecular processes III," Berichte der Bunsengesellschaft für physikalische Chemie, vol. 79, no. 2, pp. 170-183, 1975.

${ }^{36}$ M. Bartolomei, E. Carmona-Novillo, M. I. Hernández, J. Campos-Martínez, and R. Moszynski, "Global ab initio potential energy surface for the $\mathrm{O}_{2}\left({ }^{3} \Sigma_{g}^{-}\right)+\mathrm{N}_{2}\left({ }^{1} \Sigma_{g}^{+}\right)$interaction. applications to the collisional, spectroscopic, and thermodynamic properties of the complex," The Journal of Physical Chemistry A, vol. 118, no. 33, pp. 6584-6594, 2014.

${ }^{37}$ E. Garcia, A. Kurnosov, A. Lagana, F. Pirani, M. Bartolomei, and M. Cacciatore, "Efficiency of collisional $\mathrm{O}_{2}+\mathrm{N}_{2}$ vibrational energy exchange," The Journal of Physical Chemistry B, 2015.

${ }^{38}$ D. White, "Shock-tube study of vibrational exchange in $\mathrm{N}_{2}-\mathrm{O}_{2}$ mixtures," The Journal of Chemical Physics, vol. 49, no. 12 , pp. $5472-5476,1968$.

${ }^{39}$ T. Slanger, L. Jusinski, G. Black, and G. Gadd, "A new laboratory source of ozone and its potential atmospheric implications," Science, vol. 241, no. 4868, pp. 945-950, 1988.

${ }^{40}$ F. R. Gilmore, E. Bauer, and J. W. McGowan, "A review of atomic and molecular excitation mechanisms in nonequilibrium gases up to 20000 K," Journal of Quantitative Spectroscopy and Radiative Transfer, vol. 9, no. 2, pp. 157-183, 1969.

${ }^{41}$ D. R. White and R. C. Millikan, "Vibrational relaxation in air," AIAA Journal, vol. 2, no. 10, pp. 1844-1846, 1964.

${ }^{42}$ I. Ulusoy, D. Andrienko, I. Boyd, and R. Hernandez, "Quantum and quasi-classical collisional dynamics of $\mathrm{O}_{2}-\mathrm{Ar}$ at high temperatures," The Journal of Chemical Physics, submitted.

43 R. C. Millikan and D. R. White, "Systematics of vibrational relaxation," Journal of Chemical Physics, vol. 39, no. 12, pp. 3209-3213, 1963.

${ }^{44}$ N. Generalov and S. Losev, "Vibrational exitation and decomposition of molecular oxygen and carbon dioxide behind shock waves," Journal of Quantum Spectroscopy and Radiative Transfer, vol. 6, pp. 101-125, 1966.

${ }^{45}$ K. L. Wray, "Chemical kinetics of high temperature air," tech. rep., Avco-Everett Research Laboratory, Avco Corporation, 1961.

${ }^{46}$ L. Jerig, K. Thielen, and P. Roth, "High-temperature dissociation of oxygen diluted in argon or nitrogen," AIAA journal, vol. 29, no. 7, pp. 1136-1139, 1991.

${ }^{47}$ I. V. Adamovich, S. O. MacHeret, J. W. Rich, and C. E. Treanor, "Vibrational energy transfer rates using a forced harmonic oscillator model," Journal of Thermophysics and Heat Transfer, vol. 12, no. 1, pp. 57-65, 1998.

${ }^{48}$ J. G. Kim and I. D. Boyd, "Thermochemical nonequilibirum modeling of electronically excited molecular oxygen," in 11th AIAA/ASME Joint Thermophysics and Heat Transfer Conference, AIAA paper 2014-2963, June 2014. 
This article has been cited by:

1. Daniil Andrienko, Iain D. Boyd. Dissociation of oxygen and nitrogen in a bimolecular reaction at hypersonic temperatures . [Citation] [PDF] [PDF Plus]

2. Daniil A. Andrienko, Iain D. Boyd. 2017. State-specific dissociation in O 2 -O 2 collisions by quasiclassical trajectory method. Chemical Physics 491, 74-81. [Crossref]

3. Daniil Andrienko, Iain D. Boyd. Simulation of oxygen dissociation on a six-dimensional O4 potential energy surface . [Citation] [PDF] [PDF Plus] 